\title{
Beamforming Design and Performance Analysis of Full- Duplex Cooperative NOMA Systems
}

DOI:

10.1109/TWC.2019.2913425

\section{Document Version}

Accepted author manuscript

Link to publication record in Manchester Research Explorer

\section{Citation for published version (APA):}

Mobini, Z., Mohammadi, M., Chalise, B. K., Suraweera, H. A., \& Ding, Z. (2019). Beamforming Design and Performance Analysis of Full-Duplex Cooperative NOMA Systems. IEEE Transactions on Wireless

Communications, 18(6), 3295-3311. https://doi.org/10.1109/TWC.2019.2913425

\section{Published in:}

IEEE Transactions on Wireless Communications

\section{Citing this paper}

Please note that where the full-text provided on Manchester Research Explorer is the Author Accepted Manuscript or Proof version this may differ from the final Published version. If citing, it is advised that you check and use the publisher's definitive version.

\section{General rights}

Copyright and moral rights for the publications made accessible in the Research Explorer are retained by the authors and/or other copyright owners and it is a condition of accessing publications that users recognise and abide by the legal requirements associated with these rights.

\section{Takedown policy}

If you believe that this document breaches copyright please refer to the University of Manchester's Takedown Procedures [http://man.ac.uk/04Y6Bo] or contact uml.scholarlycommunications@manchester.ac.uk providing relevant details, so we can investigate your claim.

\section{OPEN ACCESS}




\title{
Beamforming Design and Performance Analysis of Full-Duplex Cooperative NOMA Systems
}

\author{
Zahra Mobini, Member, IEEE, Mohammadali Mohammadi, Member, IEEE, \\ Batu K. Chalise, Senior Member, IEEE, Himal A. Suraweera, Senior Member, IEEE, \\ and Zhiguo Ding, Senior Member, IEEE
}

\begin{abstract}
We consider downlink non-orthogonal multiple access transmission where an access point communicates with a set of near and far users via a full-duplex multiple antenna relay. To deal with the inter-user interference at the near user and selfinterference at the relay, we propose optimum and suboptimal beamforming schemes. In addition, we consider two different user selection criteria, namely: 1) random near user and random far user (RNRF) selection; 2) nearest near user and nearest far user (NNNF) selection, and derive the outage probabilities of the near and far users. Our findings reveal that as compared to half-duplex operation, full-duplex relaying can reduce the outage probability of the near users up to $63 \%$ in the case of NNNF user selection. With suboptimal beamforming schemes, the NNNF user selection shows a superior performance as compared to the RNRF user selection for all choices of transmit power, while with the optimum beamforming, the performance of RNRF user selection converges to the NNNF user selection at high transmit power. Simulation results are provided to confirm the accuracy of the developed analytical results and facilitate a better performance comparison.
\end{abstract}

\section{INTRODUCTION}

The spectral efficiency of future fifth generation (5G) systems is expected to significantly increase as compared to the fourth generation (4G) mobile communication systems. To this end, non-orthogonal multiple access (NOMA) has been recognized as a promising technology to achieve high spectral efficiency. According to the principle of NOMA, by exploiting the power domain, multiple users are multiplexed simultaneously to use the same radio resources [2]. Therefore, NOMA deviates from current orthogonal multiple access (OMA) techniques that allocate one resource block exclusively

Manuscript received Nov. 30, 2017; revised Aug. 08, 2018 and Jan. 01, 2019; accepted Apr. 21, 2019. The work of Z. Ding was supported by the UK EPSRC under grant number EP/L025272/2 and by H2020-MSCA-RISE2015 under grant number 690750. This paper was presented in part at the IEEE Global Communications Conference (GLOBECOM 2017), Singapore, Dec. 2017 [1]. The associate editor coordinating the review of this paper and approving it for publication was Prof. R. K. Ganti.(Corresponding author: Zahra Mobini.)

Z. Mobini and M. Mohammadi are with the Faculty of Engineering, Shahrekord University, Shahrekord 115, Iran (e-mail: \{z.mobini, m.a.mohammadi\}@sku.ac.ir).

B. K. Chalise is with Department of Electrical and Computer Engineering, New York Institute of Technology, Northern Boulevard, Old Westbury, NY 11568 (email: batu.k.chalise@ieee.org).

H. A. Suraweera is with the Department of Electrical and Electronic Engineering, University of Peradeniya, Peradeniya 20400, Sri Lanka (email: himal@ee.pdn.ac.lk).

Z. Ding is with School of Electrical and Electronic Engineering, the University of Manchester, Manchester, the UK (email: zhiguo.ding@manchester.ac.uk). to serve a user. In NOMA systems, multiplexing several users on the same frequency channel causes multiuser interference (MUI) which must be removed with the help of sophisticated successive interference cancellation (SIC) receivers. There is already a sizable body of literature on the theory and practical aspects of NOMA systems, where the compatibility of NOMA with other 5G key technologies such as multiple-input multiple-out (MIMO) transmission has been highlighted [3].

On a parallel development, in-band full-duplex operation has recently received significant attention, because of its capability to double the spectral efficiency of traditional halfduplex relaying [4]. Although full-duplex radars have been around since the 1940s, the self-interference (SI) problem is considered as one of the key challenges encountered in the design of full-duplex communication systems. A full-duplex transceiver can transmit and receive simultaneously in the same frequency band. Therefore, to implement full-duplex transmission at a transceiver, SI due to its own transmission to the incoming signal must be mitigated [5]. Today, passive cancellation methods, e.g., placement of radio frequency (RF) absorbers, use of wavetraps, directional antennas etc., complemented by active analog and digital cancellation stages, have been proposed to effectively suppress the SI [6]. Moreover, if full-duplex terminals are empowered with multiple antennas or massive arrays, spatial mitigation techniques can be used to further control the harmful effects of SI [5], [7]. Therefore, SI can be canceled to an acceptable level, and the practical implementation of full-duplex transceivers in modern communication systems will soon become a reality.

An ongoing main challenge for NOMA networks is that the co-existence of the near and far users results in a performance degradation for the far users [3], [8]. The performance of these networks however, can be further improved by using user cooperation [8]-[10] or dedicated relays [1], [11]-[22]. In user-assisted cooperative NOMA, a user with a better channel conditions, also referred to as the near user, helps the far user which is likely to experience a poor connection to the access point (AP) since the former is able to decode the desired information and the information intended for the latter [8]. In relay-assisted NOMA systems, a dedicated relay is employed to assist the far user [11]. There has been a growing body of research that investigates the design of relay-assisted NOMA systems. In [11], a dedicated relay has been used to design a multiuser MIMO cooperative NOMA system with better outage performance. In [12], the exact and asymptotic expressions for the average rates of a relay- 
assisted NOMA system over Rayleigh fading channels have been developed. The capacity scaling law of a NOMA system with coordinated direct and decode-and-forward (DF) relay transmission has been derived in [13]. Amplify-and-forward relay-assisted NOMA transmission of [14] has been shown to achieve a superior coding gain as compared to a cooperative OMA strategy. In [15], a detection scheme that can be applied in relay-assisted NOMA to achieve significant performance gains has been proposed. The work in [16] has considered NOMA performance for a scenario where two DF relays are used to help source-destination transmission. A two relay NOMA model has also been studied in [17] where the relays either apply dirty paper coding or use time division multiple access to serve two users. Relay selection is a popular technique considered in the present literature to combat fading and reduce the system complexity. In the context of cooperative NOMA, different relay selection criteria have been considered in [18] and [19] and these existing studies show that increasing the number of cooperative relays helps to improve the performance significantly. In [20] and [21], the resource allocation and relay beamforming schemes for the relay-assisted NOMA, capable of significantly outperforming OMA schemes, have been studied. Several works have also studied the performance of the relay-assisted NOMA in specific application scenarios such as simultaneous wireless information and power transfer [22].

Common to all of the above works [8]-[22] is the halfduplex operation assumption at the relaying node. On the other hand, the complementary nature of NOMA and fullduplex can be combined to satisfy the high spectral efficiency requirements of 5G and beyond communications [23], [24]. However, full-duplex cooperative NOMA transmission introduces several challenges such as SI due to signal leakage from the relay's output to the input and inter-user interference at the near user [24]. In [25], a full-duplex device-to-device aided cooperative NOMA scheme was proposed, where the full-duplex near user assists the base station transmissions to the far user. In [26], a full-duplex relay-assisted cooperative NOMA scheme with dual-users was examined. It was shown that the proposed full-duplex relay-assisted NOMA system in [26] achieves better performance than the half-duplex one in the low to medium signal-to-noise ratio (SNR) regime. The authors in [27] provided the diversity analysis of a hybrid full-duplex/half-duplex user-assisted NOMA system with two users. In [28], the performance of a full-duplex NOMA system is investigated, where uplink and downlink NOMA transmissions are simultaneously carried out.

In this paper, unlike references [25]-[28] that have analyzed two-user full-duplex NOMA systems with and without singleantenna relay, we study the performance. of a full-duplex multiple antenna relay-assisted NOMA system. The multiple antenna assumption allows us to study the NOMA performance with different beamforming designs and achieve spatial domain SI suppression at the relay. Moreover, we employ stochastic geometry for modeling the locations of the users and include a user selection scheme into our system model. Similar to [10], the users close to the AP are grouped together while the users near to the cell edge form another group.
In particular, we consider two groups of users: near users, randomly deployed within a disc, and far users, randomly deployed within a ring, where their respective locations are modeled as homogeneous Poisson point processes (PPPs). In addition, we employ the concept of opportunistic scheduling which is effective in improving the performance of multiuser networks [29]. Accordingly, we assume that the AP communicates with only one selected near user and one far user with the assistance of one selected relay and consider the following user selection strategies, namely (i) random near user and random far user (RNRF) selection and (ii) nearest near user and nearest far user (NNNF) selection [10]. In this paper, we focus on beamforming design and performance analysis and leave other sophisticated user selection strategies which may further improve the performance as a future research direction.

We employ suboptimum beamforming methods such as maximum ratio combining (MRC), maximal ratio transmission (MRT), and zero-forcing (ZF) at the relay, to obtain receive and transmit beamformers which mitigate the SI effect. Moreover, the beamformer optimization problem is formulated and solved using an efficient approach.

The main contributions of this work are as follows:

- We consider both inter-user interference at the near user and SI at the full-duplex relay and derive the outage probabilities of the RNRF and NNNF user selection strategies, when several suboptimum beamformers are applied at the relay. In order to highlight the system behavior and provide important insights into the performance, closed-form upper and lower bounds on the outage probability as well as simple expressions valid for certain special cases are also presented. These studies reveal the effects of key system parameters, such as the number of relay antennas; the strength of the residual SI and residual inter-user interference; user zone and density on the system performance. A key observation is that the proposed suboptimum beamforming schemes achieve the same outage performance for the near users. However, they provide different tradeoffs among the system performance, complexity, and user fairness.

- An optimum receiver and transmit relay beamformer design, based on the semidefinite relaxation (SDR) approach, is proposed, where the objective is to maximize the signal-to-interference-plus-noise ratio (SINR) at the near user while guaranteeing that the SINR at the far user is above a certain value. Our results show that with the suboptimum designs, the NNNF user selection scheme achieves superior SINR performance compared with RNRF in all the transmit power regimes. From analysis based on single-antenna systems, it has been understood that NNNF performs better than RNRF in almost all cases [10]. However, with the help of optimum beamforming and for high transmit power regime, we find that the performance of RNRF can be as good as NNNF. This is a promising result since RNRF can be implemented without knowledge of CSI and provides greater fairness than NNNF.

- Our findings reveal that the full-duplex relaying can reduce the outage probability of the near users up to 
$63 \%$ in the case of NNNF user selection and up to $55 \%$ in the case of RNRF user selection as compared to the half-duplex relaying. In addition, increasing the number of transmit antennas significantly improves the far user outage performance of the MRC/ZF beamforming design, while the outage performance of the ZF/MRT design is slightly improved by increasing the number of receive antennas. Interestingly, simulation results show that the impact of particular beamforming design on the outage performance of the far users is more significant for the NNNF user selection than that for the RNRF user selection. Also, the MRC/MRT scheme outperforms other suboptimal designs for scenarios in which the radius of the far user's zone is large.

Notation: We use bold upper case letters to denote matrices, bold lower case letters to denote vectors. The superscripts $(\cdot)^{*}$, $(\cdot)^{T}$, and $(\cdot)^{\dagger}$ stand for conjugate, transpose, and conjugate transpose, respectively; $\mathbb{E}\{x\}$ denotes the expectation of the random variable $x$; the Euclidean norm of the vector and the trace are denoted by $\|\cdot\|$, and $\operatorname{tr}(\cdot)$, respectively; $\mathcal{C N}\left(\mu, \sigma^{2}\right)$ denotes a circular symmetric complex Gaussian random variable (RV) with mean $\mu$ and variance $\sigma^{2} ; \Gamma(a)$ is the Gamma function; $\Gamma(a, x)$ is upper incomplete Gamma function [30, Eq. (8.350)].

\section{SYSTEM MODEL}

Consider a network with an AP and two groups of randomly deployed users: near and far users as shown in Fig. 1. The near users $\left\{U_{1, i}\right\}, i=1, \cdots, N_{U_{1}}$, are deployed within a disc of radius $R_{1}$, denoted by $D_{n}$, and the far users $\left\{U_{2, i}\right\}$, $i=1, \cdots, N_{U_{2}}$, are deployed within a ring of inner and outer radii $R_{2}$ and $R_{3}{ }^{1}$. denoted by $D_{f}$, In order to make ensure that the performance analysis for the far users is tractable, we assume that $R_{2} \gg R_{1}$. The locations of the near and far users are modeled according to PPPs $\Phi_{n}$ and $\Phi_{f}$, respectively, with the densities $\lambda_{n}$ and $\lambda_{f}$. We focus on the downlink NOMA transmission with one near user and one far user. Specifically, in this system set up, there is a direct link between the AP and near user $U_{1, i}$ while such a link does not exist between the AP and the far user $U_{2, i}$ [13], [26]. In order to assist far user communications, we exploit $K$ full-duplex DF relays, $\left\{\mathbb{R}_{k}\right\}, k=1, \cdots, K$, symmetrically deployed at a distance $R_{1}$ from the cell center in a circular fashion, that forward the information to the far users. Randomness of the relay locations might provide further performance improvements at the expense of increasing system implementation complexity. Hence, our model assumes deterministic deployment of the relays [32], whereas random deployment is left as a future research direction.

We assume a single-antenna AP communication aided by the infrastructure-based relays where each relay is equipped with $N_{\mathrm{R}}$ antennas for reception and $N_{\mathrm{T}}$ antennas for transmission. This model with a single antenna AP facilities system

\footnotetext{
${ }^{1}$ Once values for $R_{1}$ and $R_{2}$ are decided for performance optimization, intermediate users that neither fall into the near user nor far user categories could be served using OMA resources [10] since the use of NOMA resources for the intermediate users will not significantly enhance the spectral efficiency, compared to that of OMA [31].
}

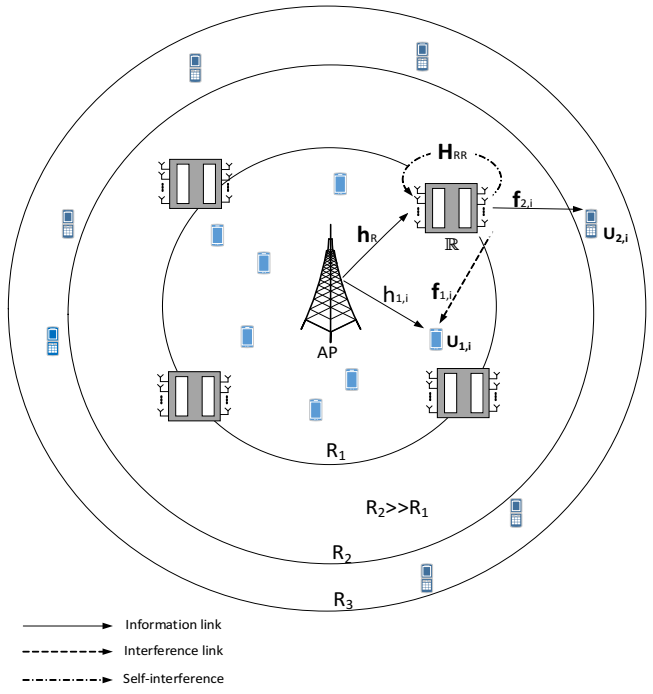

Fig. 1. The considered downlink NOMA system model with relay-assisted transmission, wherein $U_{1, i}$ and $U_{2, i}$ are the selected near user and selected far user, respectively, $\mathbb{R}$ is the selected FD relay, and $\mathbf{H}_{R R}$ and $\mathbf{f}_{1, i}$ are the residual SI and inter-user interference channels, respectively.

analysis and the derived expressions are useful to obtain design insights. Moreover, in the considered NOMA downlink transmission, the signal is processed through a single $\mathrm{RF}$ chain and transmitted from the AP antenna. Also, signal reception at the users is performed using a single antenna and a receive RF chain. For a more realistic propagation model, we assume that the links experience both large-scale path loss effects and small-scale fading. Rayleigh distributed channel coefficients are approximately constant over an observation time, $T$, (corresponding to the channel coherence time) and vary independently between different slots. As appropriate, we define the distance $d_{\sharp \#}$ between node $\sharp \in\left\{A P, \mathbb{R}_{k}\right\}$ and $\# \in\left\{U_{1, i}, U_{2, i}, \mathbb{R}_{k}\right\}$. The bounded path loss model $\ell(\sharp, \#)=\frac{\beta_{0}}{1+d_{\sharp \#}^{\alpha}}$ between node $\sharp$ and $\#$ is used, which guarantees that the path loss is always greater than one even if $d_{\sharp \#}<1$, where $\alpha \geq 2$ denotes the path loss exponent, and $\beta_{0}=\left(\frac{c}{4 \pi f_{c}}\right)^{2}$, denotes the free space path loss at a transmitter-receiver separation distance of $1 \mathrm{~m}$ at the carrier frequency, $f_{c}$ [33], [34]. For notational convenience, if node $\sharp$ is the AP located at the origin, the index $\sharp$ will be omitted, i.e., $\ell(A P, \#)=\ell(\#)$ and $d_{A P \#}=d_{\#}$. Before transmission, two users $U_{1, i}$ and $U_{2, i}$ are selected to perform NOMA transmission with the aid of the selected relay, denoted by $\mathbb{R}$, where the selection criterion for user selection and relay selection will be discussed in Subsection II-B.

\section{A. Transmission Protocol}

According to the NOMA concept [2], the AP transmits a combination of messages to both users and the selected relay $\mathbb{R}$ as

$$
s[n]=\sqrt{P_{S} a_{1, i}} x_{1, i}[n]+\sqrt{P_{S} a_{2, i}} x_{2, i}[n],
$$

where $P_{S}$ is the AP transmit power and $x_{k, i}, k \in\{1,2\}$ denotes the information symbol to $U_{k, i}$, and $a_{k, i}$ denotes the power allocation coefficient, such that $a_{1, i}+a_{2, i}=1$ and 
$a_{1, i}<a_{2, i}$. Since the selected relay $\mathbb{R}$ operates in the fullduplex mode, it simultaneously receives $s[n]$ and forwards $r[n]$ with power $P_{R}$ to the $U_{2, i}$. The received signal at $\mathbb{R}$ can be expressed as ${ }^{2}$.

$$
y_{R}[n]=\sqrt{\ell(\mathbb{R})} \mathbf{h}_{R} s[n]+\mathbf{H}_{R R} r[n]+\mathbf{n}_{R}[n],
$$

where we model the $N_{R} \times N_{T}$ residual SI channel $\mathbf{H}_{R R}$ as identically independent distributed (i.i.d) $\mathcal{C N}\left(0, \sigma_{R R}^{2}\right) \mathrm{RVs}$ [5], [6], $\mathbf{h}_{R} \in \mathcal{C}^{N_{\mathrm{R}} \times 1}$ is the channel between the AP and $\mathbb{R}$ and its entries are i.i.d, $\mathcal{C N}(0,1), \mathbf{n}_{R}[n]$ is the additive white Gaussian noise (AWGN) at the relay with $\mathbb{E}\left\{\mathbf{n}_{R} \mathbf{n}_{R}^{\dagger}\right\}=\sigma_{R}^{2} \mathbf{I}$, and $r[n]$ is the transmitted relay signal satisfying $\mathbb{E}\left\{r[n] r^{\dagger}[n]\right\}=P_{R}$, given by

$$
r[n]=\sqrt{P_{R}} \mathbf{w}_{t, i} x_{2, i}[n-\delta],
$$

where $\delta$ accounts for the time delay caused by relay processing [5]. Since the relay $\mathbb{R}$ adopts the DF protocol, upon receiving the signal, it first applies a linear combining vector $\mathbf{w}_{r}$ on $y_{R}$ to obtain an estimate of $s[n]$, denoted by $\hat{s}[n]$, as

$$
\hat{s}[n]=\sqrt{\ell(\mathbb{R})} \mathbf{w}_{r}^{\dagger} \mathbf{h}_{R} s[n]+\mathbf{w}_{r}^{\dagger} \mathbf{H}_{R R} r[n]+\mathbf{w}_{r}^{\dagger} \mathbf{n}_{R}[n] .
$$

Next the relay decodes the information intended for $U_{2, i}$ while treating the symbol of $U_{1, i}$ as interference [26]. Finally, the relay forwards $x_{2, i}[n-\delta]$ to $U_{2, i}$ using the transmit beamforming vector $\mathbf{w}_{t, i}$. Let $\left\|\mathbf{w}_{t, i}\right\|^{2}=\left\|\mathbf{w}_{r}\right\|^{2}=1$. The received SINR at the selected relay $\mathbb{R}$ is given by

$$
\gamma_{R}=\frac{P_{S} a_{2, i} \ell(\mathbb{R})\left|\mathbf{w}_{r}^{\dagger} \mathbf{h}_{R}\right|^{2}}{P_{S} a_{1, i} \ell(\mathbb{R})\left|\mathbf{w}_{r}^{\dagger} \mathbf{h}_{R}\right|^{2}+P_{R}\left|\mathbf{w}_{r}^{\dagger} \mathbf{H}_{R R} \mathbf{w}_{t, i}\right|^{2}+\sigma_{R}^{2}} .
$$

On the other hand, the received signal at $U_{1, i}$ can be written as

$$
\begin{aligned}
y_{1, i}[n]= & \sqrt{\ell\left(U_{1, i}\right)} h_{1, i} s[n]+\sqrt{P_{R} \ell\left(\mathbb{R}, U_{1, i}\right)} \mathbf{f}_{1, i}^{T} \mathbf{w}_{t, i} x_{2, i}[n-\delta] \\
& +n_{1, i}[n],
\end{aligned}
$$

where $h_{1, i} \sim \mathcal{C N}(0,1)$ is the channel between the AP and $U_{1, i}, \mathbf{f}_{1, i} \in \mathcal{C}^{N_{T} \times 1}$ denotes the channel between the relay and $U_{1, i}$, and $n_{1, i}[n] \sim \mathcal{C N}\left(0, \sigma_{n_{1}}^{2}\right)$ denotes the AWGN at the $U_{1, i}$. Moreover, $\ell\left(\mathbb{R}, U_{1, i}\right)=\frac{1}{1+d_{\mathbb{R} U_{1, i}}^{\alpha}}$ with $d_{\mathbb{R} U_{1, i}}=$ $\sqrt{R_{1}^{2}+d_{U_{1, i}}^{2}-2 R_{1} d_{U_{1, i}} \cos \left(\theta_{r}-\theta_{i}\right)}$, where $\theta_{r}$ denotes the angle of the selected relay $\mathbb{R}$ from reference $\mathrm{x}$-axis and $\theta_{i}$ denotes the angle of the $U_{1, i}$ from reference $\mathrm{x}$-axis, $-\pi \leq$ $\theta_{r}-\theta_{i} \leq \pi$.

It is assumed that $x_{2, i}[n-\delta]$ is known to $U_{1, i}$, and thus $U_{1, i}$ can remove it via interference cancellation [26]. Nevertheless, here, we consider the case of imperfect interference cancellation wherein $U_{1, i}$ cannot perfectly remove $x_{2, i}[n-\delta]$. In particular, we model the elements of the $N_{T} \times 1$ channel $\mathbf{f}_{1, i}$, known as the inter-user interference channel, as i.i.d $\mathcal{C N}\left(0, q_{r} \times 1\right)$ RVs, where $q_{r}$ represents the strength of the inter-user interference [26]. Specifically, $q_{r}=0$ implies perfect interference cancellation at $U_{1, i}$.

\footnotetext{
${ }^{2}$ In practice, ideal SI cancellation is impossible to achieve since transmi distortion noise due to front-end hardware imperfections is not perfectly known [5]. Accordingly, in our transmission protocol, we consider the effect of residual SI
}

Applying the principle of NOMA concept, SIC is carried out at $U_{1, i}$. In particular, $U_{1, i}$ first decodes the message of $U_{2, i}$, i.e., $x_{2, i}$, then subtracts it from the received signal to detect its own message, if $x_{2, i}$ is decoded correctly. Therefore, the received SINR at $U_{1, i}$ to detect $x_{2, i}$ of $U_{2, i}$ is given by

$$
\gamma_{1, i}^{x_{2, i}}=\frac{P_{S} a_{2, i} \ell\left(U_{1, i}\right)\left|h_{1, i}\right|^{2}}{P_{S} a_{1, i} \ell\left(U_{1, i}\right)\left|h_{1, i}\right|^{2}+P_{R} \ell\left(\mathbb{R}, U_{1, i}\right)\left|\mathbf{f}_{1, i}^{T} \mathbf{w}_{t, i}\right|^{2}+\sigma_{n_{1}}^{2}},
$$

and the received SINR at $U_{1, i}$ to detect its own message, $x_{1, i}$, is given by

$$
\gamma_{1, i}^{x_{1, i}}=\frac{P_{S} a_{1, i} \ell\left(U_{1, i}\right)\left|h_{1, i}\right|^{2}}{P_{R} \ell\left(\mathbb{R}, U_{1, i}\right)\left|\mathbf{f}_{1, i}^{T} \mathbf{w}_{t, i}\right|^{2}+\sigma_{n_{1}}^{2}} .
$$

Finally, the observation at $U_{2, i}$ can be expressed as follows:

$$
y_{2, i}[n]=\sqrt{P_{R} \ell\left(\mathbb{R}, U_{2, i}\right)} \mathbf{f}_{2, i}^{T} \mathbf{w}_{t, i} x_{2, i}[n-\delta]+n_{2, i}[n],
$$

where $\ell\left(\mathbb{R}, U_{2, i}\right)=\frac{1}{1+d_{\mathbb{R} U_{2, i}}^{\alpha}} \quad$ with $\quad d_{\mathbb{R} U_{2, i}}=$ $\sqrt{R_{1}^{2}+d_{U_{2, i}}^{2}-2 R_{1} d_{U_{2, i}} \cos \left(\theta_{r}-\dot{\theta}_{i}\right)}, \quad \dot{\theta}_{i}$ denotes the angle of $U_{2, i}$ from reference $\mathrm{x}$-axis, $\mathbf{f}_{2, i} \in \mathcal{C}^{N_{\mathrm{T}} \times 1}$ denotes the channel between $\mathbb{R}$ and $U_{2, i}$ and $n_{2, i}[n] \sim \mathcal{C N}\left(0, \sigma_{n_{2}}^{2}\right)$ denotes the AWGN at $U_{2, i}$. Therefore, the received SNR at $U_{2, i}$ is given by

$$
\gamma_{2, i}^{x_{2, i}}=\frac{P_{R} \ell\left(\mathbb{R}, U_{2, i}\right)\left|\mathbf{f}_{2, i}^{T} \mathbf{w}_{t, i}\right|^{2}}{\sigma_{n_{2}}^{2}}
$$

\section{B. User Selection and Relay Selection Strategies}

The NOMA principle can be implemented in two ways [3]. One way is to order the users according to their channel conditions, which assumes that there are no strict qualityof-service (QoS) requirements. The second approach is to order the users according to their QoS requirements, instead of their channel conditions. In this paper, we consider the first way of NOMA implementation which assumes that the users do not have strict QoS requirements and can be served opportunistically using the RNRF and NNNF strategies. In particular, for the RNRF strategy, the AP randomly selects the near user $U_{1, i}$ and the far user $U_{2, i}$ from the two groups of users. For the NNNF strategy, a user within the disc, $D_{n}$, with the shortest distance to the AP is selected as a near user ${ }^{3} U_{1, i}^{\star}$ and the user within ring, $D_{f}$, with the shortest distance to the AP is selected as a far user $U_{2, i}^{\star}$. It is worth pointing out that the considered user selection strategies yield different tradeoffs among system complexity, reliability, and user fairness. For example, RNRF does not need to know the users' channel information for performing the user selection strategy, which reduces the system overhead. NNNF tries to pair the nearest near user and the nearest far user for NOMA, which yields the best performance due to small path loss but might result in potential issues in user fairness.

For each user selection strategy, the relay with the minimum Euclidean distance from the selected far user is chosen for

\footnotetext{
${ }^{3}$ Here after, superscript " $\star$ " is used to indicate the selected near user, selected far user, and the corresponding outage probabilities with the NNNF user selection strategy.
} 
cooperative NOMA. We can define the relay selection criterion as

$$
\min \left\{\left\|\mathbb{R}_{k}, U_{2, i}\right\|, k \in\{1, \cdots, K\}\right\}
$$

This relay selection strategy is suitable for practical scenarios, wherein the far users are much farther away from the AP in comparison with the near users, and thus have the poor channel conditions. Accordingly, the criterion in (11) can improve the reception reliability of the far users.

\section{Full-DUPleX COOPERATIVE NOMA WITH RNRF USER SELECTION}

In this section, we characterize the system performance with the RNRF user selection. Its implementation does not require the knowledge of the instantaneous CSI of the users. From (5), (7), (8), and (10), it is evident that the received SINR and SNR of both the near and far users are dependent on the beamforming design at the selected relay $\mathbb{R}$. Hence, in the sequel we adopt three beamforming designs described in the literature [35], [36], namely transmit ZF (TZF), receive ZF (RZF), and MRC/MRT.

Case 1) TZF Scheme: If the selected relay is equipped with $N_{\mathrm{T}}>1$ transmit antennas, SI can be canceled out by projecting the transmit signal to the null space of the received signal at the relay input [35]. Furthermore, we fix the MRC beamforming vector $\mathbf{w}_{r}^{\mathrm{MRC}}=\frac{\mathbf{h}_{R}}{\left\|\mathbf{h}_{R}\right\|}$ at the relay receiver. Therefore, the optimal transmit beamforming vector $\mathbf{w}_{t, i}$ is obtained by solving the following problem:

$$
\begin{aligned}
\max _{\left\|\mathbf{w}_{t, i}\right\|=1} & \left|\mathbf{f}_{2, i}^{T} \mathbf{w}_{t, i}\right|^{2} \\
\text { s.t. } & \mathbf{h}_{R}^{\dagger} \mathbf{H}_{R R} \mathbf{w}_{t, i}=0 .
\end{aligned}
$$

Using similar steps as in [35], the optimal transmit vector $\mathbf{w}_{t, i}$ in (12) is obtained as $\mathbf{w}_{t, i}^{\mathrm{ZF}}=\frac{\mathbf{A f}_{2, i}^{*}}{\left\|\mathbf{A f}_{2, i}^{*}\right\|}$, where $\mathbf{A}=\mathbf{I}_{N_{\mathrm{T}}}-$ $\frac{\mathbf{H}_{R R}^{\dagger} \mathbf{h}_{R} \mathbf{h}_{R}^{\dagger} \mathbf{H}_{R R}}{\left\|\mathbf{h}_{R}^{\dagger} \mathbf{H}_{R R}\right\|^{2}}$.

Case 2) RZF Scheme: As a second scheme, we assume that $\mathbf{w}_{t, i}^{\mathrm{MRT}}=\frac{\mathbf{f}_{2, i}^{*}}{\left\|\mathbf{f}_{2, i}\right\|}$, i.e., the relay employees the MRT beamforming vector, and uses $\mathrm{ZF}$ criterion for designing the receive beamforming vector $\mathbf{w}_{r}$. When the selected relay is equipped with $N_{\mathrm{R}}>1$ receive antennas, the undesired SI can be completely nullified. In this case, the optimization of $\mathbf{w}_{r}$ can be expressed as [35]

$$
\begin{aligned}
\max _{\left\|\mathbf{w}_{r}\right\|=1} & \left|\mathbf{w}_{r}^{\dagger} \mathbf{h}_{R}\right|^{2}, \\
\text { s.t. } & \mathbf{w}_{r}^{\dagger} \mathbf{H}_{R R} \mathbf{f}_{2, i}^{*}=0 .
\end{aligned}
$$

The optimal solution of (13), $\mathbf{w}_{r}^{\mathrm{ZF}}$, can be expressed as $\mathbf{w}_{r}^{\mathrm{ZF}}=$ $\frac{\mathbf{B h}_{R}}{\left\|\mathbf{B h}_{R}\right\|}$, where $\mathbf{B}=\mathbf{I}_{N_{\mathrm{R}}}-\frac{\mathbf{H}_{R R} \mathbf{f}_{2, i}^{*} \mathbf{f}_{2, i}^{T} \mathbf{H}_{R R}^{\dagger}}{\left\|\mathbf{H}_{R R} \mathbf{f}_{2, i}^{*}\right\|^{2}}$.

Case 3) MRC/MRT Scheme: The MRC/MRT scheme is applied in half-duplex relay-assisted systems, and hence it is interesting to investigate the performance of the full-duplex relay-assisted NOMA system with the MRC/MRT scheme. Specifically, the receive and transmit beamformers are selected as $\mathbf{w}_{r}^{\mathrm{MRC}}=\frac{\mathbf{h}_{R}}{\left\|\mathbf{h}_{R}\right\|}$ and $\mathbf{w}_{t, i}^{\mathrm{MRT}}=\frac{\mathbf{f}_{2, i}^{*}}{\left\|\mathbf{f}_{2, i}\right\|}$, respectively.

\section{A. Outage Probability of the Near Users}

An outage event at the near user $U_{1, i}$ occurs when $x_{2, i}$ is decoded in error or when $x_{2, i}$ is decoded correctly but $x_{1, i}$ is decoded in error. Let $\tau_{1}=2^{\mathcal{R}_{1}}-1$ and $\tau_{2}=2^{\mathcal{R}_{2}}-1$, where $\mathcal{R}_{1}$ and $\mathcal{R}_{2}$ are the transmission rates at $U_{1, i}$ and $U_{2, i}$, respectively. The outage probability at $U_{1, i}$ can be expressed as [26]

$$
\mathrm{P}_{\text {out }, 1}=1-\operatorname{Pr}\left(\gamma_{1, i}^{x_{2, i}}>\tau_{2}, \gamma_{1, i}^{x_{1, i}}>\tau_{1}\right) .
$$

1) TZF Scheme: Substituting $\mathrm{w}_{r}^{\mathrm{MRC}}$ and $\mathrm{w}_{t, i}^{\mathrm{ZF}}$ into (7) and (8), the received SINR at $U_{1, i}$ to detect $x_{2, i}$ with TZF, $\tilde{\gamma}_{1, i}^{x_{2, i}}$, and the received SINR at $U_{1, i}$ to detect $x_{1, i}$ with $\mathrm{TZF}, \tilde{\gamma}_{1, i}^{x_{1, i}}$, can be obtained as

$$
\tilde{\gamma}_{1, i}^{x_{2, i}}=\frac{P_{S} a_{2, i} \ell\left(U_{1, i}\right)\left|h_{1, i}\right|^{2}}{P_{S} a_{1, i} \ell\left(U_{1, i}\right)\left|h_{1, i}\right|^{2}+P_{R} \ell\left(\mathbb{R}, U_{1, i}\right)\left|\mathbf{f}_{1, i}^{T} \mathbf{w}_{t, i}^{\mathrm{ZF}}\right|^{2}+\sigma_{n_{1}}^{2}},
$$

and

$$
\tilde{\gamma}_{1, i}^{x_{1, i}}=\frac{P_{S} a_{1, i} \ell\left(U_{1, i}\right)\left|h_{1, i}\right|^{2}}{P_{R} \ell\left(\mathbb{R}, U_{1, i}\right)\left|\mathbf{f}_{1, i}^{T} \mathbf{w}_{t, i}^{\mathrm{ZF}}\right|^{2}+\sigma_{n_{1}}^{2}},
$$

respectively. Accordingly, based on (14), the following proposition presents the outage probability of $U_{1, i}$ with the TZF scheme.

Proposition 1. The outage probability of $U_{1, i}$ with the TZF scheme is given by

$$
\begin{aligned}
\mathrm{P}_{\text {out }, 1}^{\mathrm{TZF}}=1 & -\frac{1}{\pi R_{1}^{2}} \int_{0}^{R_{1}} \int_{-\pi}^{\pi} \frac{e^{-\mu\left(1+r^{\alpha}\right)}}{1+\frac{q_{r} \rho_{r} \mu\left(1+r^{\alpha}\right)}{1+\left(R_{1}^{2}+r^{2}-2 r R_{1} \cos \left(\theta_{r}-\theta_{i}\right)\right)^{\frac{\alpha}{2}}}} \\
& \times r d \theta_{i} d r
\end{aligned}
$$

if $\tau_{2} \leq \frac{a_{2, i}}{a_{1, i}}$, otherwise $\mathrm{P}_{\text {out }, 1}^{\mathrm{TZF}}=1$, where $\mu=\max \left(\frac{1}{\zeta}, \frac{\tau_{1}}{\rho_{s} a_{1, i}}\right)$ with $\zeta=\frac{\rho_{s} a_{2, i}-\rho_{s} a_{1, i} \tau_{2}}{\tau_{2}}, \rho_{s}=\frac{P_{S}}{N_{0}}, \rho_{r}=\frac{P_{R}}{N_{0}}$, and $N_{0}$ is the mean power of noise at the near user ${ }^{4}$.

Proof: See Appendix A.

From (17), we see that the outage probability of the near users with RNRF is independent of the users density, $\lambda_{n}$. This is because RNRF selects users randomly, and hence increasing the number of near users will not affect its performance.

In order to derive approximate closed-form expressions, we now set $\cos \left(\theta_{r}-\theta_{i}\right)= \pm 1$. In particular, by setting $\cos \left(\theta_{r}-\theta_{i}\right)=+1, \ell\left(\mathbb{R}, U_{1, i}\right)$ is maximized, and hence the inter-user interference at $U_{1, i}$ is maximized, which minimizes $\gamma_{1, i}^{x_{1, i}}$ and $\gamma_{1, i}^{x_{2, i}}$. On the other hand, $\cos \left(\theta_{r}-\theta_{i}\right)=-1$ results in the minimum inter-user interference at $U_{1, i}$. Consequently, from (17), the upper bound on the outage probability of $U_{1, i}$ can be written as

$$
\mathrm{P}_{\text {out }, 1}^{\mathrm{TZF}, \mathrm{U}}=1-\frac{2}{R_{1}^{2}} \int_{0}^{R_{1}} \frac{e^{-\mu\left(1+r^{\alpha}\right)}}{1+\frac{q_{r} \rho_{r} \mu\left(1+r^{\alpha}\right)}{1+\left(R_{1}^{2}+r^{2}-2 \eta R_{1} r\right)^{\frac{\alpha}{2}}}} r d r,
$$

where $\eta=1$ ( $\eta=-1$ for the lower bound). To the best of our knowledge, the integral in (18) does not admit a closed-form solution, however by following a similar approach as in [10],

\footnotetext{
${ }^{4}$ Without lost of generality, it is assumed that the mean power of noise at all users and relay is the same and denoted by $N_{0}$.
} 
we use the Gaussian-Chebyshev quadrature method [37] to obtain

$$
\mathrm{P}_{\text {out }, 1}^{\mathrm{TZF}, \mathrm{U}} \approx 1-\frac{\pi}{2 M} \sum_{m=1}^{M} \frac{\sqrt{\left(1-\phi_{m}\right)\left(1+\phi_{m}\right)^{3}}}{1+\frac{q_{r} \rho_{r} \mu\left(1+c_{m}^{\alpha}\right)}{1+\left(R_{1}^{2}+c_{m}^{2}-2 \eta R_{1} c_{m}\right)^{\frac{\alpha}{2}}}} e^{-\mu\left(1+c_{m}^{\alpha}\right)},
$$

where $c_{m}=\left(\phi_{m}+1\right) \frac{R_{1}}{2}, \phi_{m}=\cos \left(\frac{2 m-1}{2 M} \pi\right)$ and $M$ is a parameter to guarantee a desirable complexity-accuracy tradeoff. This expression explicitly shows that the outage performance of the near users with the RNRF selection is jointly determined by four factors: 1) the strength of the interuser interference, $\left.q_{r}, 2\right)$ the AP and relay transmission powers, 3 ) the path loss exponent, and 4) the radius of the near user's disc, $R_{1}$. Additionally, the outage performance of the near users with TZF is independent of the number of antennas at the relay.

Now, to obtain additional insights on the outage performance, we consider a full-duplex cooperative NOMA scenario with perfect inter-user interference cancellation at $U_{1, i}$, i.e., $q_{r}=0$. Substituting $q_{r}=0$ in (59), the outage probability of $U_{1, i}$ with the TZF scheme can be written as

$$
\mathrm{P}_{\text {out }, 1}^{\mathrm{TZF}, \mathrm{P}}=1-\frac{2}{R_{1}^{2}} \int_{0}^{R_{1}} e^{-\mu\left(1+r^{\alpha}\right)} r d r .
$$

For an arbitrary choice of $\alpha$, the integral in (20) is mathematically intractable, and hence we use the Gaussian-Chebyshev quadrature method. Therefore, (20) can be approximately expressed in closed-form as

$$
\mathrm{P}_{\text {out }, 1}^{\mathrm{TZF}, \mathrm{P}} \approx 1-\frac{\pi}{2 M} \sum_{m=1}^{M} \sqrt{\left(1-\phi_{m}\right)\left(1+\phi_{m}\right)^{3}} e^{-\mu\left(1+c_{m}^{\alpha}\right)} .
$$

As an immediate observation from (21), we see that the outage performance for the near users improves with decreasing $R_{1}$, smaller path loss, and higher source transmission power. Moreover, for the special case of $\alpha=2, \mathrm{P}_{\text {out, } 1}^{\text {TZF,P }}$ can be obtained from (20) as an exact expression which is given by

$$
\mathrm{P}_{\text {out }, 1}^{\top Z F, P}=\left\{\begin{array}{cl}
1-\frac{e^{-\mu}}{\mu R_{1}^{2}}+\frac{e^{-\mu\left(1+R_{1}^{2}\right)}}{\mu R_{1}^{2}}, & \tau_{2} \leq \frac{a_{2}}{a_{1}} \\
1, & \tau_{2}>\frac{a_{2}}{a_{1}}
\end{array}\right.
$$

which presents the lowest possible theoretical lower bound on the outage probability of the near users among communication scenarios with different values of $\alpha$, namely, $2 \leq \alpha \leq 6$.

2) $R Z F$ Scheme: Substituting $\mathbf{w}_{t, i}^{\mathrm{MRT}}$ into (7) and (8), the received SINR at $U_{1, i}$ to detect $x_{2, i}$ with RZF, $\hat{\gamma}_{1, i}^{x_{2, i}}$, and the received SINR at $U_{1, i}$ to detect $x_{2, i}$ with RZF, $\hat{\gamma}_{1, i}^{x_{1, i}}$, can be obtained as

$$
\hat{\gamma}_{1, i}^{x_{2, i}}=\frac{P_{S} a_{2, i} \ell\left(U_{1, i}\right)\left|h_{1, i}\right|^{2}}{P_{S} a_{1, i} \ell\left(U_{1, i}\right)\left|h_{1, i}\right|^{2}+P_{R} \ell\left(\mathbb{R}, U_{1, i}\right)\left|\mathbf{f}_{1, i}^{T} \mathbf{w}_{t, i}^{\mathrm{MRT}}\right|^{2}+\sigma_{n_{1}}^{2}},
$$

and

$$
\hat{\gamma}_{1, i}^{x_{1, i}}=\frac{P_{S} a_{1, i} \ell\left(U_{1, i}\right)\left|h_{1, i}\right|^{2}}{P_{R} \ell\left(\mathbb{R}, U_{1, i}\right)\left|\mathbf{f}_{1, i}^{T} \mathbf{w}_{t, i}^{\mathrm{MRT}}\right|^{2}+\sigma_{n_{1}}^{2}},
$$

respectively.

From (15), (16), (23), and (24) $\left|\mathbf{f}_{1, i}^{T} \mathbf{w}_{t, i}^{\mathrm{ZF}}\right|^{2}$ and $\left|\mathbf{f}_{1, i}^{T} \mathbf{w}_{t, i}^{\mathrm{MRT}}\right|^{2}$ are exponential RVs with the same mean $q_{r}$, and hence $\hat{\gamma}_{1, i}^{x_{1, i}}$ and $\hat{\gamma}_{1, i}^{x_{2, i}}$ have the same statistical characteristics as $\tilde{\gamma}_{1, i}^{x_{1, i}}$ and $\tilde{\gamma}_{1, i}^{x_{2, i}}$, respectively. Accordingly, based on (14), we get $\mathrm{P}_{\text {out }, 1}^{\mathrm{TZF}}=\mathrm{P}_{\text {out }, 1}^{\mathrm{RZF}}$. Additionally, the presented results for the outage probability of $U_{1, i}$ with the TZF scheme are identical for that of the RZF counterpart.

3) $M R C / M R T$ Scheme: From (7) and (8), we observe that the received SINR at the near user is dependent only on $\mathbf{w}_{t, i}$. Since both the RZF and MRC/MRT schemes use the same transmit beamformer $\mathbf{w}_{t, i}^{\mathrm{MRT}}$, we have $\mathrm{P}_{\text {out }, 1}^{\mathrm{MRC}}=\mathrm{P}_{\text {out }, 1}^{\mathrm{RZF}}=\mathrm{P}_{\text {out }, 1}^{\mathrm{TZF}}$.

We see that all of the proposed beamforming schemes achieve the same outage performance for the near users. However, as studied below, the proposed beamforming schemes provide different performance/complexity tradeoffs for the far users.

\section{B. Outage Probability of the Far Users}

The outage event at $U_{2, i}$ is due to the following two cases: 1) $\mathbb{R}$ cannot decode $x_{2, i}$, and 2) $\mathbb{R}$ can decode $x_{2, i}$ but $x_{2, i}$ cannot be decoded correctly by $U_{2, i}$. Therefore, the outage probability at $U_{2, i}$ can be written as

$$
P_{\text {out }, 2}=\operatorname{Pr}\left(\gamma_{R}<\tau_{2}\right)+\operatorname{Pr}\left(\gamma_{R}>\tau_{2}\right) \operatorname{Pr}\left(\gamma_{2, i}^{x_{2, i}}<\tau_{2}\right) .
$$

1) $T Z F$ Scheme: Applying $\mathrm{w}_{r}^{\mathrm{MRC}}$ and $\mathrm{w}_{t, i}^{\mathrm{ZF}}$ into (5) and (10), the received SINR at the relay with TZF, $\tilde{\gamma}_{R}$, and the received SNR at $U_{2, i}$ with TZF, $\tilde{\gamma}_{2, i}^{x_{2, i}}$, can be obtained, respectively. The following proposition presents the outage probability of the TZF scheme for an arbitrary choice of $\alpha$.

Proposition 2. The outage probability of $U_{2, i}$ with the TZF scheme is given by

$$
\begin{aligned}
& \mathrm{P}_{\text {out }, 2}^{\mathrm{TZF}}=1-\frac{\pi}{M\left(R_{3}+R_{2}\right) \Gamma\left(N_{\mathrm{R}}\right)} \Gamma\left(N_{\mathrm{R}}, \frac{\left(1+R_{1}^{\alpha}\right)}{\zeta}\right) \sum_{k=0}^{N_{\mathrm{T}}-2} \frac{1}{k !} \\
& \times\left(\frac{\tau_{2}}{\rho_{r}}\right)^{k} \sum_{m=1}^{M} z_{m} \sqrt{1-\phi_{m}^{2}}\left(1+z_{m}^{\alpha}\right)^{k} e^{-\left(\frac{\tau_{2}}{\rho_{r}}\right)\left(1+z_{m}^{\alpha}\right)},
\end{aligned}
$$

where $z_{m}=\frac{R_{3}-R_{2}}{2}\left(\phi_{m}+1\right)+R_{2}$.

Proof: See Appendix B.

We observe that $\mathrm{P}_{\text {out,2 }}^{T Z F}$ depends on the number of receive/transmit antennas, the far user's zone, the transmission power, and the path loss. In particular, $\mathrm{P}_{\text {out }, 2}^{\mathrm{TZF}}$ is decreasing with $P_{S}, P_{R}$, and the number of receive/transmit antennas. However, from (19) and Proposition 1, as $P_{R}$ increases, the inter-user interference increases and the outage probability of the near users increases. Thus, one can improve the outage performance of the far users by increasing the number of transmit antennas without deteriorating the outage performance of the near users.

Note that in an interference-limited network, the SNR distribution can be replaced by the SIR distribution in (25) to obtain a much simpler analytical expression. For example, when noise is ignored, $P_{\text {out } 2}^{\text {TZF }}$ in (25) can be written as

$$
\begin{aligned}
\mathrm{P}_{\text {out }, 2}^{\mathrm{TZF}}= & \operatorname{Pr}\left(\frac{a_{2, i}}{a_{1, i}}<\tau_{2}\right)+\operatorname{Pr}\left(\frac{a_{2, i}}{a_{1, i}}>\tau_{2}\right) \\
& \times \operatorname{Pr}\left(\rho_{r} \ell\left(\mathbb{R}, U_{2, i}\right) Y_{3}<\tau_{2}\right),
\end{aligned}
$$


in which, to guarantee the implementation of NOMA, the condition $\frac{a_{2, i}}{a_{1, i}} \geq \tau_{2}$ should be satisfied, and thus $\operatorname{Pr}\left(\frac{a_{2, i}}{a_{1, i}}<\tau_{2}\right)=$ 0 . Accordingly, $\mathrm{P}_{\text {out }, 2}^{\mathrm{TZF}}$ can be written as

$$
\begin{aligned}
\mathrm{P}_{\text {out }, 2}^{\text {TZF }} \approx & \operatorname{Pr}\left(\rho_{r} \ell\left(U_{2, i}\right) Y_{3}<\tau_{2}\right) \\
\approx & 1-\frac{\pi}{M\left(R_{3}+R_{2}\right)} \sum_{k=0}^{N_{\mathrm{T}}-2} \frac{1}{k !}\left(\frac{\tau_{2}}{\rho_{r}}\right)^{k} \sum_{m=1}^{M} z_{m} \sqrt{1-\phi_{m}^{2}} \\
& \times\left(1+z_{m}^{\alpha}\right)^{k} e^{-\left(\frac{\tau_{2}}{\rho_{r}}\right)\left(1+z_{m}^{\alpha}\right)} .
\end{aligned}
$$

Clearly (28) is independent of $P_{S}$ and $N_{R}$. Therefore, in an interference-limited network, increasing the source transmit power and the number of receive antennas does not increase the outage performance. We now turn our attention towards characterizing the outage probability of the far users for the special case of $\alpha=2$ in the interference-limited regime. By applying $\alpha=2$ in (27), and then using the integral identity of [30, Eq. (2.33.11)], we obtain

$$
\mathrm{P}_{\text {out }, 2}^{\mathrm{TZF}}=1-\frac{1}{R_{3}^{2}-R_{2}^{2}} \sum_{k=0}^{N_{\mathrm{T}}-2}\left(\frac{\tau_{2}}{\rho_{r}}\right)^{k}\left(G\left(R_{2}\right)-G\left(R_{3}\right)\right),
$$

where $G(x)=e^{-\left(\frac{\tau_{2}}{\rho_{r}}\right)\left(1+x^{2}\right)} \sum_{j=0}^{k} \frac{\left(1+x^{2}\right)^{j}}{j !}\left(\frac{\tau_{2}}{\rho_{r}}\right)^{j-k-1} \cdot \mathrm{We}$ see that the outage performance depends on the radius of the far user's zone.

2) RZF Scheme: Applying $\mathrm{w}_{r}^{\mathrm{ZF}}$ and $\mathrm{w}_{t, i}^{\mathrm{MRT}}$ into (5) and (10), the received SINR at the relay with RZF, $\hat{\gamma}_{R}$, and the received SNR at $U_{2, i}$ with RZF, $\hat{\gamma}_{2, i}^{x_{2}, i}$, can be obtained, respectively. Using the outage definition in (25) and similar to (26), we can derive the outage probability of the far users with the RZF scheme as:

$$
\begin{gathered}
\mathrm{P}_{\text {out }, 2}^{\mathrm{RZF}}=1-\frac{\pi}{M\left(R_{3}+R_{2}\right) \Gamma\left(N_{\mathrm{R}}-1\right)} \Gamma\left(N_{\mathrm{R}}-1, \frac{\left(1+R_{1}^{\alpha}\right)}{\zeta}\right) \\
\times \sum_{k=0}^{N_{\mathrm{T}}-1} \frac{1}{k !}\left(\frac{\tau_{2}}{\rho_{r}}\right)^{k} \sum_{m=1}^{M} z_{m} \sqrt{1-\phi_{m}^{2}}\left(1+z_{m}^{\alpha}\right)^{k} e^{-\left(\frac{\tau_{2}}{\rho_{r}}\right)\left(1+z_{m}^{\alpha}\right)} .
\end{gathered}
$$

Based on (26) and (30), it is clear that the TZF and RZF schemes exhibit the same outage probability of the far users for some antenna configurations. For example, if we consider the values of $N_{T}$ and $N_{R}$ as a pair $\left(N_{T}, N_{R}\right), \operatorname{TZF}\left(N_{T}, N_{R}\right)$ has the same outage performance with RZF $\left(N_{T}-1, N_{R}+1\right)$. Moreover, for both the TZF and RZF schemes, the outage performance of the far users is an increasing function of $P_{S}$ and $P_{R}$ due to the fact that the receive/transmit $\mathrm{ZF}$ operation completely cancels the SI at the relay's input/output and as a result, increasing $P_{R}$ improves the second-hop SNR of the far users. In the case of the MRC/MRT scheme, this behavior is somewhat different. On the other hand, as we observed from (17), the outage probability of the near users is decreasing with $P_{S}$ and is increasing with $P_{R}$. Therefore, to further enhance the performance of relay-assisted NOMA transmissions, it is important to optimally allocate total power between the AP and relay, and jointly optimize the receive/transmit beamformers of the relay.
3) MRC/MRT Scheme: Substituting $\mathbf{w}_{r}^{\mathrm{MRC}}$ and $\mathrm{w}_{t, i}^{\mathrm{MRT}}$ into (5) and (10), the received SINR at the relay and the received SNR at $U_{2, i}$ with the MRC/MRT scheme can be obtained, respectively. The following proposition provides the outage probability of $U_{2, i}$.

Proposition 3. The outage probability of $U_{2, i}$ with the MRC/MRT scheme is given by (31) at the top of the next page.

Proof: See Appendix C.

As evident in Subsection III-A, the outage probability of the near users for the proposed beamforming schemes is independent of the number of antennas at the relay. However, it is interesting to study the outage performance of the far users when $N_{\mathrm{R}}$ and $N_{\mathrm{T}}$ grow large. Using the law of large numbers and the results presented in [7], we can show that when $N_{\mathrm{R}} \rightarrow \infty$ and $N_{\mathrm{T}} \rightarrow \infty$, the outage probabilities for the three proposed beamforming schemes with RNRF user selection can be further simplified as

$$
\mathrm{P}_{\text {out }, 2} \approx\left\{\begin{array}{cl}
0, & \frac{\rho_{r} N_{\mathrm{T}}}{\tau_{2}}>R_{3}^{\alpha}+1, \\
\frac{R_{3}^{2}-\left(\frac{\rho_{r} N_{\mathrm{T}}}{\tau_{2}}-1\right)^{\frac{2}{\alpha}}}{R_{3}^{2}-R_{2}^{2}}, & R_{2}^{\alpha}+1<\frac{\rho_{r} N_{\mathrm{T}}}{\tau_{2}}<R_{3}^{\alpha}+1, \\
1, & \frac{\rho_{r} N_{\mathrm{T}}}{\tau_{2}}<R_{2}^{\alpha}+1 .
\end{array}\right.
$$

\section{Half-Duplex Relaying}

Let us now consider the half-duplex operation for a relayassisted cooperative NOMA transmission. The system model is the similar to that of the full-duplex counterpart, except that two time slots are used for the reception and transmission at the relay, respectively. Specifically, for a transmission block time of $T, \frac{T}{2}$ is dedicated to the AP for transmitting a combination of messages to both users and the selected relay and the remaining $\frac{T}{2}$ is used by the relay for transmitting information to the far users. Accordingly, the received SNR at $\mathbb{R}$ can be expressed as

$$
\varsigma_{R}=\frac{P_{S} a_{2, i} \ell(\mathbb{R})\left|\mathbf{w}_{r}^{\dagger} \mathbf{h}_{R}\right|^{2}}{P_{S} a_{1, i} \ell(\mathbb{R})\left|\mathbf{w}_{r}^{\dagger} \mathbf{h}_{R}\right|^{2}+\sigma_{R}^{2}} .
$$

In addition, the received SINRs at $U_{1, i}$ to detect $x_{2, i}$ and to detect $x_{1, i}$ are, respectively, given by

$$
\varsigma_{1, i}^{x_{2, i}}=\frac{P_{S} a_{2, i} \ell\left(U_{1, i}\right)\left|h_{1, i}\right|^{2}}{P_{S} a_{1, i} \ell\left(U_{1, i}\right)\left|h_{1, i}\right|^{2}+\sigma_{n_{1}}^{2}},
$$

and

$$
\varsigma_{1, i}^{x_{1, i}}=\frac{P_{S} a_{1, i} \ell\left(U_{1, i}\right)\left|h_{1, i}\right|^{2}}{\sigma_{n_{1}}^{2}} .
$$

Moreover, the received SNR at $U_{2, i}, \varsigma_{2, i}^{x_{2, i}}$, is given by (10). Let $\tau_{1}^{\mathrm{HD}}=2^{2 \mathcal{R}_{1}}-1$ and $\tau_{2}^{\mathrm{HD}}=2^{2 \mathcal{R}_{2}}-1$. Considering MRC/MRT as the receive/transmit beamformers, in the next proposition, we present the outage probability expressions for the near and far users with half-duplex relaying.

Proposition 4. The outage probabilities of $U_{1, i}$ and $U_{2, i}$ with the half-duplex relaying are given by

$$
\mathrm{P}_{\text {out }, 1}^{\mathrm{HD}} \approx 1-\frac{\pi}{2 M} \sum_{m=1}^{M} \sqrt{\left(1-\phi_{m}\right)\left(1+\phi_{m}\right)^{3}} e^{-\mu^{\mathrm{HD}}\left(1+c_{m}^{\alpha}\right)},
$$




$$
\begin{aligned}
\mathrm{P}_{\mathrm{out}, 2}^{\mathrm{MRC}} & =1-\frac{\pi}{M\left(R_{3}+R_{2}\right)} \sum_{k=0}^{N_{\mathrm{T}}-1} \frac{1}{k !}\left(\frac{\tau_{2}}{\rho_{r}}\right)^{k} \sum_{m=1}^{M} z_{m} \sqrt{1-\phi_{m}^{2}}\left(1+z_{m}^{\alpha}\right)^{k} e^{-\left(\frac{\tau_{2}}{\rho_{r}}\right)\left(1+z_{m}^{\alpha}\right)} \\
& \times\left(\frac{1}{\Gamma\left(N_{R}\right)} \Gamma\left(N_{R}, \frac{1+R_{1}^{\alpha}}{\zeta}\right)-\frac{e^{\frac{1}{\rho_{r} \sigma_{R R}^{2}}}}{\Gamma\left(N_{R}\right)}\left(\frac{\zeta}{\rho_{r} \sigma_{R R}^{2}\left(1+R_{1}^{\alpha}\right)}+1\right)^{-N_{R}} \Gamma\left(N_{R}, \frac{1}{\rho_{r} \sigma_{R R}^{2}}+\frac{1+R_{1}^{\alpha}}{\zeta}\right)\right) .
\end{aligned}
$$

and

$$
\begin{aligned}
& \mathrm{P}_{\text {out }, 2}^{\mathrm{HD}}=1-\frac{\pi}{M\left(R_{3}+R_{2}\right) \Gamma\left(N_{\mathrm{R}}\right)} \Gamma\left(N_{\mathrm{R}}, \frac{1+R_{1}^{\alpha}}{\zeta_{\mathrm{HD}}^{\alpha}}\right)^{N_{\mathrm{T}}-1} \sum_{k=0} \frac{1}{k !} \\
& \left(\frac{\tau_{2}^{\mathrm{HD}}}{\rho_{r}}\right)^{k} \sum_{m=1}^{M} z_{m} \sqrt{1-\phi_{m}^{2}}\left(1+z_{m}^{\alpha}\right)^{k} e^{-\left(\frac{\tau_{2}^{\mathrm{HD}}}{\rho_{r}}\right)\left(1+z_{m}^{\alpha}\right)}
\end{aligned}
$$

respectively, where $\mu^{\mathrm{HD}}=\max \left(\frac{1}{\zeta^{\mathrm{HD}}}, \frac{\tau_{1}^{\mathrm{HD}}}{\rho_{s} a_{1, i}}\right)$ with $\zeta^{\mathrm{HD}}=$ $\frac{\rho_{s} a_{2, i}-\rho_{s} a_{1, i} \tau_{2}^{\mathrm{HD}}}{\tau_{2}^{\mathrm{HD}}}$.

Proof: See Appendix D.

From (36), we see that, the outage performance of the near user $\mathrm{P}_{\text {out }, 1}^{\mathrm{HD}}$ increases with decreasing $R_{1}$ and it is independent of $P_{R}$, which is in contrast to the full-duplex operation. This result is intuitively expected because under half-duplex operation, the AP and relay transmit in two different time slots and the near users do not suffer from the inter-user interference, and also with the reduced $R_{1}$, path loss is reduced. From (37), it can be observed that increasing $P_{R}$ increases the outage performance of the far users.

\section{Full-duplex CoOperative NOMA With NNNF USER SELECTION}

In this section, we investigate the outage performance of the NNNF user selection scheme, in which the users' CSI is utilized to select the near and far users with the shortest distance to the AP. Accordingly, the NNNF user selection can minimize the outage probability of both the near and far users.

\section{A. Outage Probability of the Near Users}

1) TZF Scheme: By invoking (14), we can study the outage probability of the near users. We have the following key result:

Proposition 5. The outage probability of $U_{1, i}^{\star}$ with the TZF scheme is given by

$$
\begin{aligned}
\mathrm{P}_{\text {out }, 1^{\star}}^{\mathrm{TZF}}=1 & -\frac{v_{n}}{2 \pi} \int_{0}^{R_{1}} \int_{-\pi}^{\pi} \frac{e^{-\mu\left(1+r^{\alpha}\right)}}{1+\frac{q_{r} \rho_{r} \mu\left(1+r^{\alpha}\right)}{1+\left(R_{1}^{2}+r^{2}-2 r R_{1} \cos \left(\theta_{r}-\theta_{i}\right)\right)^{\frac{\alpha}{2}}}} \\
& \times r e^{-\pi \lambda_{n} r^{2}} d \theta_{i} d r,
\end{aligned}
$$

where $v_{n}=\frac{2 \pi \lambda_{n}}{1-e^{-\pi \lambda_{n} R_{1}^{2}}}$.

Proof: See Appendix E.

The main difference between the RNRF and the NNNF strategies is that the outage probability for NNNF is dependent on the density of the near users. In particular, $\mathrm{P}_{\text {out }, 1^{\star}}^{\mathrm{TZF}}$ is a function of both the design parameters $R_{1}$ and $\lambda_{n}$, whereas $\mathrm{P}_{\text {out }, 1}^{\mathrm{TZF}}$ is only influenced by $R_{1}$. We next focus on a few special cases and/or asymptotic results which yield closedform expressions.

Similar to the RNRF strategy, the outage probability, $\mathrm{P}_{\text {out }, 1^{\star}}^{\mathrm{TZF}, \mathrm{U}}$, can be upper bounded $(\eta=1)$ and lower bounded $(\eta=-1)$ as

$$
\begin{aligned}
\mathrm{P}_{\mathrm{out}, 1^{\star}}^{\mathrm{TZF}, \mathrm{U}} & \approx 1-\frac{\pi v_{n} R_{1}}{2 M} \sum_{m=1}^{M} \sqrt{\left(1-\phi_{m}^{2}\right)} \\
& \times \frac{e^{-\mu\left(1+c_{m}^{\alpha}\right)} c_{m} e^{-\pi \lambda_{n} c_{m}^{2}}}{1+\frac{q_{r} \rho_{r} \mu}{1+\left(R_{1}^{2}+c_{m}^{2}-2 \eta R_{1} c_{m}\right)^{\frac{\alpha}{2}}}\left(1+c_{m}^{\alpha}\right)} .
\end{aligned}
$$

This expression clearly shows that $P_{\text {out }, 1 *}^{T Z F}, \mathcal{U}$ decreases when the density of the near users increases. Additionally, the outage probability of $U_{1, i}^{\star}$ of NNNF with the TZF scheme and perfect inter-user interference cancellation at $U_{1, i}^{\star}$ can be expressed in closed-form, for an arbitrary $\alpha$, as

$$
\begin{aligned}
& \mathrm{P}_{\text {out }, 1^{\star}}^{\mathrm{TZF}, \mathrm{P}}=1-v_{n} \int_{0}^{R_{1}} e^{-\mu\left(1+r^{\alpha}\right)} r e^{-\pi \lambda_{n} r^{2}} d r \\
& \approx 1-\frac{\pi v_{n} R_{1}}{2 M} \sum_{m=1}^{M} \sqrt{\left(1-\phi_{m}^{2}\right)} e^{-\mu\left(1+c_{m}^{\alpha}\right)} c_{m} e^{-\pi \lambda_{n} c_{m}^{2}} .
\end{aligned}
$$

For the special case of $\alpha=2, \mathrm{P}_{\text {out }, 1^{\star}}^{\text {TZF }}$ can be further simplified to

$$
\mathrm{P}_{\text {out }, 1^{\star}}^{\mathrm{TZF}, \mathrm{P}}= \begin{cases}1-\frac{v_{n}\left(e^{-\mu}-e^{-R_{1}^{2}\left(\mu+\pi \lambda_{n}\right)-\mu}\right)}{2\left(\mu+\pi \lambda_{n}\right)} & \tau_{2} \leq \frac{a_{2, i}}{a_{1, i}}, \\ 1 & \tau_{2}>\frac{a_{2, i}}{a_{1, i}} .\end{cases}
$$

From (41), as $\lambda_{n} \rightarrow \infty$, we have $\mathrm{P}_{\text {out, } 1^{\star}}^{\mathrm{TZF}} \sim 1-e^{-\mu}$ which is independent of $\lambda_{n}$ and $R_{1}$, and decreases exponentially with $P_{S}$.

2) RZF Scheme: $\hat{\gamma}_{1, i}^{x_{1, i}}$ and $\hat{\gamma}_{1, i}^{x_{2, i}}$ have the same statistical characteristics as $\tilde{\gamma}_{1, i}^{x_{1, i}}$ and $\tilde{\gamma}_{1, i}^{x_{2, i}}$, respectively, and thus the results presented in (38), (39), (40), and (41) also hold for the RZF scheme.

3) MRC/MRT Scheme: Both the RZF and MRC/MRT schemes use the same transmit beamformer $\mathrm{w}_{t, i}^{\mathrm{MRC}}$, and accordingly the presented results for the TZF and RZF schemes are identical for the MRC/MRT scheme.

\section{B. Outage Probability of the Far Users}

1) TZF scheme: Using the definition in (25), we analyze the outage probability of the far users. The following proposition presents the outage probability valid for an arbitrary $\alpha$.

Proposition 6. The outage probability of $U_{2, i}^{\star}$ with the TZF scheme is given by 


$$
\begin{aligned}
\mathrm{P}_{\text {out }, 2^{\star}}^{\mathrm{TZF}} \approx & 1-\frac{v_{f} \pi\left(R_{3}-R_{2}\right) e^{\pi \lambda_{f} R_{2}^{2}}}{2 M \Gamma\left(N_{\mathrm{R}}\right)} \Gamma\left(N_{\mathrm{R}}, \frac{\left(1+R_{1}^{\alpha}\right)}{\zeta}\right) \\
& \times \sum_{k=0}^{N_{\mathrm{T}}-2} \frac{1}{k !}\left(\frac{\tau_{2}}{\rho_{r}}\right)^{k} \sum_{m=1}^{M} z_{m} \sqrt{1-\phi_{m}^{2}}\left(1+z_{m}^{\alpha}\right)^{k} \\
& \times e^{-\left(\frac{\tau_{2}}{\rho_{r}}+\frac{\tau_{2}}{\rho_{r}} z_{m}^{\alpha}+\pi \lambda_{f} z_{m}^{2}\right)},
\end{aligned}
$$

where $v_{f}=\frac{2 \pi \lambda_{f}}{1-e^{-\pi \lambda_{f}\left(R_{3}^{2}-R_{2}^{2}\right)}}$.

Proof: See Appendix F.

We observe that $\mathrm{P}_{\text {out }, 2^{\star}}^{\mathrm{TZ}}$, similar to the outage probability of the far users with RNRF user selection, depends on the number of receive/transmit antennas, the far user's zone, the transmit powers and the path loss. In particular, $\mathrm{P}_{\text {out }, 2^{\star}}^{\mathrm{TZF}}$ is decreasing with $P_{S}, P_{R}$, and the number of receive/transmit antennas. Moreover, $\mathrm{P}_{\text {out }, 2^{\star}}^{\mathrm{TZF}}$ depends on the density of the far users, $\lambda_{f}$, while $\mathrm{P}_{\text {out, } 2}^{\mathrm{TZF}}$ is independent of $\lambda_{f}$. In the high SNR regime and for the special case of $\alpha=2$, the outage probability of $U_{2, i}^{\star}$ can be simplified to

$\mathrm{P}_{\text {out }, 2 \star}^{\mathrm{TZF}}=1-\frac{v_{f} e^{\pi \lambda_{f}\left(1+R_{2}^{2}\right)}}{2} \sum_{k=0}^{N_{\mathrm{T}}-2}\left(\frac{\tau_{2}}{\rho_{r}}\right)^{k}\left(H\left(R_{2}\right)-H\left(R_{3}\right)\right)$,

where $H(x)=e^{-\left(\frac{\tau_{2}}{\rho_{r}}+\pi \lambda_{f}\right)\left(1+x^{2}\right)} \sum_{j=0}^{k} \frac{\left(1+x^{2}\right)^{j}}{j !}\left(\frac{\tau_{2}}{\rho_{r}}+\right.$ $\left.\pi \lambda_{f}\right)^{j-k-1}$ and we have used the integral identity [30, Eq. (2.33.11)] to derive (43).

2) $R Z F$ Scheme: Based on the definition in (25) and using similar steps as in Proposition 6, the outage probability of $U_{2, i}^{\star}$ with the RZF scheme can be expressed as

$$
\begin{aligned}
\mathrm{P}_{\text {out }, 2^{\star}}^{\mathrm{RZF}} \approx 1 & -\frac{v_{f} \pi\left(R_{3}-R_{2}\right) e^{\pi \lambda_{f} R_{2}^{2}}}{2 M \Gamma\left(N_{\mathrm{R}}-1\right)} \Gamma\left(N_{\mathrm{R}}-1, \frac{\left(1+R_{1}^{\alpha}\right)}{\zeta}\right) \\
& \times \sum_{k=0}^{N_{\mathrm{T}}-1} \frac{1}{k !}\left(\frac{\tau_{2}}{\rho_{r}}\right)^{k} \sum_{m=1}^{M} z_{m} \sqrt{1-\phi_{m}^{2}}\left(1+z_{m}^{\alpha}\right)^{k} \\
& \times e^{-\left(\frac{\tau_{2}}{\rho_{r}}+\frac{\tau_{2}}{\rho_{r}} z_{m}^{\alpha}+\pi \lambda_{f} z_{m}^{2}\right)} .
\end{aligned}
$$

3) $M R C / M R T$ Scheme: Using similar steps as in Proposition 6 , the outage probability of $U_{2, i}^{\star}$ with the MRC/MRT scheme can be expressed as (45) at the top of the next page. Equations (42) and (44) indicate that $\mathrm{P}_{\text {out }}^{\mathrm{TZF}}$ (* $^{\star}$ and $\mathrm{P}_{\text {out }}^{\mathrm{RZF} 2^{\star}}$ are independent of $\sigma_{R R}^{2}$, whereas equation (45) shows that $\mathrm{P}_{\text {out, } 2^{\star}}^{\mathrm{MRC}}$ is a function of $\sigma_{R R}^{2}$. This is expected since both the TZF and RZF schemes completely eliminate the SI, while SI exists in the MRC/MRT scheme.

In the special case where $N_{\mathrm{R}} \rightarrow \infty$ and $N_{\mathrm{T}} \rightarrow \infty$, the outage probabilities of the proposed beamforming schemes with the NNNF user selection can be simplified as (46) at the top of the next page.

\section{Half-Duplex Relaying}

Let us now focus on half-duplex relaying with the NNNF user selection and MRC/MRT scheme. The outage probability of $U_{1, i}$ and $U_{2, i}$ can be derived as

$$
\begin{aligned}
\mathrm{P}_{\text {out }, 1^{\star}}^{\mathrm{HD}} & \approx 1-\frac{\pi v_{n} R_{1}}{2 M} \sum_{m=1}^{M} \sqrt{\left(1-\phi_{m}^{2}\right)} \\
& \times e^{-\mu^{\mathrm{HD}}\left(1+c_{m}^{\alpha}\right)} c_{m} e^{-\pi \lambda_{n} c_{m}^{2}}
\end{aligned}
$$

and

$$
\begin{aligned}
\mathrm{P}_{\text {out }, 2^{\star}}^{\mathrm{HD}} \approx 1 & -\frac{v_{f} \pi\left(R_{3}-R_{2}\right) e^{\pi \lambda_{f} R_{2}^{2}}}{2 M \Gamma\left(N_{\mathrm{R}}\right)} \Gamma\left(N_{\mathrm{R}}, \frac{\left(1+R_{1}^{\alpha}\right)}{\zeta^{\mathrm{HD}}}\right) \\
& \times \sum_{k=0}^{N_{\mathrm{T}-1}} \frac{1}{k !}\left(\frac{\tau_{2}^{\mathrm{HD}}}{\rho_{r}}\right)^{k} \sum_{m=1}^{M} z_{m} \sqrt{1-\phi_{m}^{2}}\left(1+z_{m}^{\alpha}\right)^{k} \\
& \times e^{-\left(\frac{\tau_{2}^{\mathrm{HD}}}{\rho_{r}}+\frac{\tau_{2}^{\mathrm{HD}}}{\rho_{r}} z_{m}^{\alpha}+\pi \lambda_{f} z_{m}^{2}\right)}
\end{aligned}
$$

respectively.

\section{OPTIMUM BEAMFORMING}

The schemes discussed in Section IV enable first-hop or second-hop SINR maximization of the far users by designing $\mathbf{w}_{r}$ or $\mathbf{w}_{t, i}$ separately when the other beamformer is fixed. In this section, we propose a method for joint optimization. Specifically, the problem of interest is to design the receive and transmit relay beamformers, $\mathbf{w}_{r}$ and $\mathbf{w}_{t, i}$, that maximize the received SINR at the near users, given a targeted SINR constraint at the far user. In particular, we consider a scenario where the near users expect to be served with the best efforts, while the far users require to reach their own quality of service (QoS) requirement [9]. The optimization problem is expressed as

$$
\begin{aligned}
\max _{\mathbf{w}_{t, i}, \mathbf{w}_{r}} & \min \left(\gamma_{1, i}^{x_{2, i}}, \gamma_{1, i}^{x_{1, i}}\right) \\
\text { s.t. } & \min \left(\gamma_{R}, \gamma_{2, i}^{x_{2, i}}\right) \geq \gamma_{t}, \\
& \left\|\mathbf{w}_{t, i}\right\|=\left\|\mathbf{w}_{r}\right\|=1,
\end{aligned}
$$

where $\gamma_{t}$ is a targeted threshold SINR required by the far user. From (7) and (8), it can be readily shown that

$$
\gamma_{1, i}^{x_{2, i}}=\frac{a_{2, i}}{a_{1, i}\left(1+\frac{1}{\gamma_{1, i}^{x_{1, i}}}\right)},
$$

which indicates that $\gamma_{1, i}^{x_{2, i}}$ can be expressed in terms of $\gamma_{1, i}^{x_{1, i}}$. Introducing an auxiliary variable $\beta \geq 0$, (49) can be expressed as

$$
\begin{aligned}
\max _{\mathbf{w}_{t, i}, \mathbf{w}_{r}, \beta} & \beta \\
\text { s.t. } & \min \left(\gamma_{1, i}^{x_{2, i}}, \gamma_{1, i}^{x_{1, i}}\right) \geq \beta \\
& \min \left(\gamma_{R}, \gamma_{2, i}^{x_{2, i}}\right) \geq \gamma_{t} \\
& \left\|\mathbf{w}_{t, i}\right\|=\left\|\mathbf{w}_{r}\right\|=1
\end{aligned}
$$

In the optimization problem (51), the constraint, $\min \left(\gamma_{1, i}^{x_{1, i}}, \gamma_{1, i}^{x_{2, i}}\right) \geq \beta$, is equivalent to the constraints, $\gamma_{1, i}^{x_{1, i}} \geq \beta$ and $\gamma_{1, i}^{x_{2, i}} \geq \beta$. Using (50), (7), and (8), these constraints can be expressed as

$$
\begin{aligned}
\left|\mathbf{f}_{1, i}^{T} \mathbf{w}_{t, i}\right|^{2} & \leq \frac{1}{\beta} \tilde{s} a_{1, i}-\tilde{r} \\
\left|\mathbf{f}_{1, i}^{T} \mathbf{w}_{t, i}\right|^{2} & \leq\left(\frac{1}{\beta} a_{2, i}-a_{1, i}\right) \tilde{s}-\tilde{r},
\end{aligned}
$$

where $\tilde{s} \triangleq \frac{P_{S} \ell\left(U_{1, i}\right)\left|h_{1, i}\right|^{2}}{P_{R} \ell\left(\mathbb{R}, U_{1, i}\right)}, \tilde{r}=\frac{\sigma_{n_{1}}^{2}}{P_{R} \ell\left(\mathbb{R}, U_{1, i}\right)}$, and $\frac{a_{2, i}}{a_{1, i}}-\beta \geq 0$. Accordingly, the optimization problem (51) can be equiva- 


$$
\begin{aligned}
\mathrm{P}_{\text {out }, 2^{\star}}^{\mathrm{MRC}} & =1-\frac{v_{f} \pi\left(R_{3}-R_{2}\right) e^{\pi \lambda_{f} R_{2}^{2}}}{2 M} \sum_{k=0}^{N_{\mathrm{T}}-1} \frac{1}{k !}\left(\frac{\tau_{2}}{\rho_{r}}\right)^{k} \sum_{m=1}^{M} z_{m} \sqrt{1-\phi_{m}^{2}}\left(1+z_{m}^{\alpha}\right)^{k} e^{-\left(\frac{\tau_{2}}{\rho_{r}}+\frac{\tau_{2}}{\rho_{r}} z_{m}^{\alpha}+\pi \lambda_{f} z_{m}^{2}\right)} \\
& \times\left(\frac{1}{\Gamma\left(N_{R}\right)} \Gamma\left(N_{R}, \frac{1+R_{1}^{\alpha}}{\zeta}\right)-\frac{e^{\frac{1}{\rho_{r} \sigma_{R R}^{2}}}}{\Gamma\left(N_{R}\right)}\left(\frac{\zeta}{\rho_{r} \sigma_{R R}^{2}\left(1+R_{1}^{\alpha}\right)}+1\right)^{-N_{R}} \Gamma\left(N_{R}, \frac{1}{\rho_{r} \sigma_{R R}^{2}}+\frac{1+R_{1}^{\alpha}}{\zeta}\right)\right) .
\end{aligned}
$$

$$
\mathrm{P}_{\text {out }, 2^{*}} \approx\left\{\begin{array}{cl}
0, & \frac{\rho_{r} N_{\mathrm{T}}}{\tau_{2}}>R_{3}^{\alpha}+1, \\
\frac{v_{f}}{2 \pi \lambda_{f}}\left(e^{-\pi \lambda_{f}\left(\left(\frac{\rho_{r} N_{\mathrm{T}}}{\tau_{2}}-1\right)^{\frac{2}{\alpha}}-R_{2}^{2}\right)}-e^{-\pi \lambda_{f}\left(R_{3}^{2}-R_{2}^{2}\right)}\right), & R_{2}^{\alpha}+1<\frac{\rho_{r} N_{\mathrm{T}}}{\tau_{2}}<R_{3}^{\alpha}+1, \\
1, & \frac{\rho_{r} N_{\mathrm{T}}}{\tau_{2}}<R_{2}^{\alpha}+1 .
\end{array}\right.
$$

lently re-expressed as

$$
\begin{aligned}
\max _{\mathbf{w}_{t, i}, \mathbf{w}_{r}, \beta} & \beta \\
\text { s.t. } & \left|\mathbf{f}_{1, i}^{T} \mathbf{w}_{t, i}\right|^{2} \leq \frac{1}{\beta} \tilde{s} a_{1, i}-\tilde{r}, \\
& \left|\mathbf{f}_{1, i}^{T} \mathbf{w}_{t, i}\right|^{2} \leq\left(\frac{1}{\beta} a_{2, i}-a_{1, i}\right) \tilde{s}-\tilde{r}, \\
& \min \left(\gamma_{R}, \gamma_{2, i}^{x_{2, i}}\right) \geq \gamma_{t}, \\
& \beta \leq \frac{a_{2, i}}{a_{1, i}}, \quad\left\|\mathbf{w}_{t, i}\right\|=\left\|\mathbf{w}_{r}\right\|=1 .
\end{aligned}
$$

In (53), only $\gamma_{R}$ depends on $\mathbf{w}_{r}$.

Obviously, for a given $\mathbf{w}_{t, i}$, the optimum $\mathbf{w}_{r}$ is the one that maximizes $\gamma_{R}$. This can be expressed as $\max _{\left\|\mathbf{w}_{r}\right\|=1} \frac{\mathbf{w}_{r}^{H} \mathbf{h}_{R} \mathbf{h}_{R}^{H} \mathbf{w}_{r}}{\mathbf{w}_{r}^{H} \mathbf{w}_{r}}$, where $\mathbf{C} \triangleq P_{S} a_{1, i} \ell(\mathbb{R}) \mathbf{h}_{R} \mathbf{h}_{R}^{H}+P_{R} \mathbf{H}_{R R} \mathbf{w}_{t, i} \mathbf{w}_{t, i}^{H} \mathbf{H}_{R R}^{H}+\sigma_{R}^{2} \mathbf{I}$. Thus, the optimum $\mathbf{w}_{r}$ is given by $\mathbf{w}_{r}=\frac{\mathbf{C}^{-1} \mathbf{h}_{R}}{\left\|\mathbf{C}^{-1} \mathbf{h}_{R}\right\|}$. Substituting this $\mathbf{w}_{r}$ into $\gamma_{R}$ and applying the Sherman-Morrison formula [38], $\gamma_{R}$ can be expressed as

$$
\begin{aligned}
& \gamma_{R}=P_{S} a_{2, i} \ell(\mathbb{R}) \mathbf{h}_{R}^{H}\left[\mathbf{D}+P_{R} \mathbf{H}_{R R} \mathbf{w}_{t, i} \mathbf{w}_{t, i}^{H} \mathbf{H}_{R R}^{H}\right]^{-1} \mathbf{h}_{R}, \\
& =P_{S} a_{2, i} \ell(\mathbb{R})\left[\mathbf{h}_{R}^{H} \mathbf{D}^{-1} \mathbf{h}_{R}-\frac{P_{R}\left|\mathbf{h}_{R}^{H} \mathbf{D}^{-1} \mathbf{H}_{R R} \mathbf{w}_{t, i}\right|^{2}}{1+P_{R} \mathbf{w}_{t, i}^{H} \mathbf{H}_{R R}^{H} \mathbf{D}^{-1} \mathbf{H}_{R R} \mathbf{w}_{t, i}}\right],(5
\end{aligned}
$$

where $\mathbf{D} \triangleq P_{S} a_{1, i} \ell(\mathbb{R}) \mathbf{h}_{R} \mathbf{h}_{R}^{H}+\sigma_{R}^{2} \mathbf{I}$. Using $\gamma_{R}$ from (54), the optimization problem (53) is expressed as

$$
\begin{array}{ll} 
& \max _{\text {s.t. }} \quad \mathbf{w}_{t, i}^{H} \mathbf{f}_{1, i}^{*} \mathbf{f}_{1, i}^{T} \mathbf{w}_{t, i} \leq \frac{1}{\beta} \tilde{s} a_{1, i}-\tilde{r}, \\
& \mathbf{w}_{t, i}^{H} \mathbf{f}_{1, i}^{*} \mathbf{f}_{1, i}^{T} \mathbf{w}_{t, i} \leq\left(\frac{1}{\beta} a_{2, i}-a_{1, i}\right) \tilde{s}-\tilde{r}, \\
& \mathbf{w}_{t, i}^{H} \mathbf{f}_{2, i}^{*} \mathbf{f}_{2, i}^{T} \mathbf{w}_{t, i} \geq d, \\
& \mathbf{w}_{t, i}^{H} \mathbf{H}_{R R}^{H} \mathbf{D}^{-1} \mathbf{h}_{R} \mathbf{h}_{R}^{H} \mathbf{D}^{-1} \mathbf{H}_{R R} \mathbf{w}_{t, i} \leq e \mathbf{w}_{t, i}^{H} \mathbf{E w}_{t, i},
\end{array}
$$

where $d \triangleq \frac{\gamma_{t} \sigma_{n_{2}}^{2}}{P_{R} \ell\left(\mathbb{R}, U_{2, i}\right)}, e \triangleq \frac{1}{P_{R}}\left[\mathbf{h}_{R}^{H} \mathbf{D}^{-1} \mathbf{h}_{R}-\frac{\gamma_{t}}{P_{S} a_{2, i} \ell(\mathbb{R})}\right]$, and $\mathbf{E} \triangleq \mathbf{I}+P_{R} \mathbf{H}_{R R}^{H} \mathbf{D}^{-1} \mathbf{H}_{R R}$. Unfortunately, the optimization problem (55) does not lead to closed-form solutions of $\mathbf{w}_{t, i}$ and $\beta$. Moreover, in its current form, (55) is not convex. However, defining auxiliary variables $\bar{\beta}$ and $\mathbf{W}_{t, i}$, where $\bar{\beta} \triangleq \frac{1}{\beta}$ and $\mathbf{W}_{t, i} \triangleq \mathbf{w}_{t, i} \mathbf{w}_{t, i}^{H}$, and then relaxing the rank-one constraint of $\mathbf{W}_{t, i}$, (55) can be expressed as the following SDR problem

$$
\begin{array}{ll} 
& \min _{t, i} \bar{\beta} \geq \frac{a_{1, i}}{a_{2, i}} \\
& \bar{\beta} \\
\text { s.t. } & \operatorname{tr}\left(\mathbf{W}_{t, i} \mathbf{f}_{1, i}^{*} \mathbf{f}_{1, i}^{T}\right) \leq \min \left(\bar{\beta} \tilde{s} a_{1, i}-\tilde{r},\left(\bar{\beta} a_{2, i}-a_{1, i}\right) \tilde{s}-\tilde{r}\right), \\
& \operatorname{tr}\left(\mathbf{W}_{t, i} \mathbf{f}_{2, i}^{*} \mathbf{f}_{2, i}^{T}\right) \geq d, \\
& \operatorname{tr}\left(\mathbf{W}_{t, i} \mathbf{H}_{R R}^{H} \mathbf{D}^{-1} \mathbf{h}_{R} \mathbf{h}_{R}^{H} \mathbf{D}^{-1} \mathbf{H}_{R R}\right) \leq e \operatorname{tr}\left(\mathbf{W}_{t, i} \mathbf{E}\right), \\
& \operatorname{tr}\left(\mathbf{W}_{t, i}\right)=1, \mathbf{W}_{t, i} \succeq 0 .
\end{array}
$$

The SDR problem (56) is in standard form. Analyzing its Karush-Kuhn-Tucker conditions and following a similar procedure as in [36], it can be shown that a rank-one optimum solution can be recovered from the solution $\mathbf{W}_{t, i}$. In this regard, the SDR problem in (56) is equivalent to the original problem (55). Then, $\mathbf{w}_{t, i}$ is simply the eigenvector corresponding to non-zero eigenvalue of $\mathbf{W}_{t, i}$.

\section{NumericAl RESUltS AND Discussion}

In this section, we present numerical results to validate our analysis, demonstrate the performance, and investigate the impact of key system parameters. The noise power spectral density is $-174 \mathrm{dBm} / \mathrm{Hz}$, the transmission bandwidth is 20 $\mathrm{MHz}, f_{c}=2.5 \mathrm{GHz}$ [39] and we assume a normalized noise power of $\frac{N_{0}}{\beta_{0}}=-50 \mathrm{dBm}$. We set $a_{1}=0.2, a_{2}=0.8, \alpha=3$, and $\mathcal{R}_{1}=\mathcal{R}_{2}=1 \mathrm{bps} / \mathrm{Hz}$ [10], [18]. Unless otherwise stated, we take $q_{r}=10 \mathrm{dBm}, \sigma_{R R}^{2}=-40 \mathrm{dBm}$, and $P_{S}=P_{R}=\frac{P}{2}$, where $P$ is the total transmit power.

\section{A. Outage Probability of the Near Users}

Fig. 2 shows the outage probability of the near users versus $P$ for the RNRF and NNNF user selection strategies, where the analytical curves are based on Propositions 1 and 5 . A close match between the analytical (solid line) and simulation (dashed line) curves can be observed. In addition, results, not shown here, confirmed that the derived outage probability bounds in (39) for the NNNF user selection are tight. This is because, in the NNNF user selection strategy, the distance of the nearest user to the AP, i.e., $d_{U_{1, i}^{\star}}$, approaches zero, and hence the term $2 R_{1} d_{U_{1, i}^{\star}} \cos \left(\theta_{r}-\theta_{i}\right)$ in 


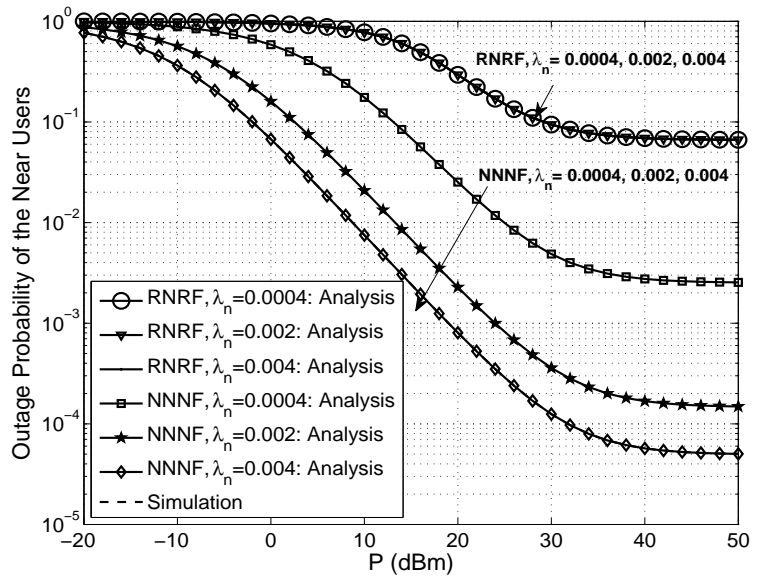

Fig. 2. Outage probability of the near users versus $P$ for the RNRF and NNNF user selection strategies with different density of the near users where $R_{1}=100 \mathrm{~m}$.

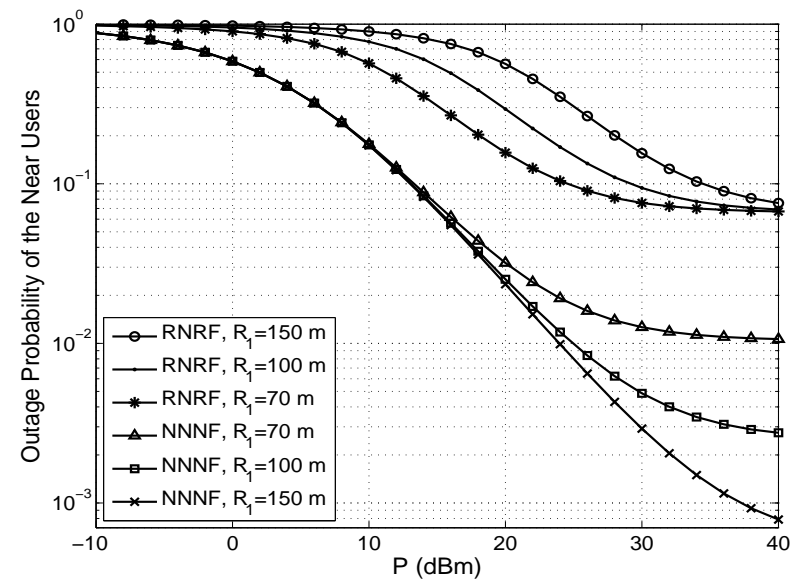

Fig. 3. Outage probability of the near users versus $P$ for different radii of the near user's disc, $R_{1}$, where $\lambda_{n}=0.0004$.

$d_{\mathbb{R}, U_{1, i}^{\star}}=\sqrt{R_{1}^{2}+d_{U_{1, i}^{\star}}^{2}-2 R_{1} d_{U_{1, i}^{\star}} \cos \left(\theta_{r}-\theta_{i}\right)}$ is small, which makes the difference between the bounds and the exact values negligible. Fig. 2 also shows that the NNNF strategy exhibits a superior outage performance in comparison to the RNRF strategy. Moreover, the outage probability of the near users with the NNNF strategy depends on the near user density $\lambda_{n}$, as elucidated in Subsection IV-A, while with the RNRF strategy, the corresponding outage probability is independent of $\lambda_{n}$. In particular, for the NNNF strategy, as the near user density $\lambda_{n}$ or the number of near users given by $\lambda_{n} \pi R_{1}^{2}$ increases, the outage probability of the near users decreases.

We investigate the impact of changing $R_{1}$ on the outage performance in Fig. 3. Increasing $R_{1}$ has two effects on the outage probability of the near users, namely, (i) increasing the path loss (a negative effect), and (ii) increasing the distance between the user and the selected relay (a positive effect). The latter effect becomes dominant under NNNF user selection, which leads to an improvement in the outage performance. Specifically, in the NNNF strategy, the nearest user to the AP is selected as the near user and increasing $R_{1}$ will not change its position notably. On the other hand, the outage performance of the near user degrades due to the interference from the relay to the near user, which decreases as $R_{1}$ is increased.

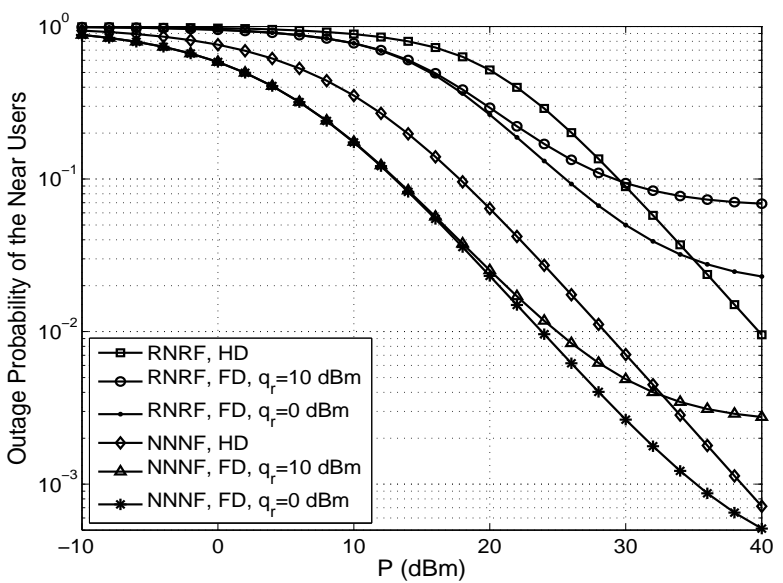

Fig. 4. Outage probability comparison between the full-duplex (FD) relaying and half-duplex (HD) relaying versus $P$ for different levels of inter-user interference strength where $R_{1}=100 \mathrm{~m}$ and $\lambda_{n}=0.0004$.

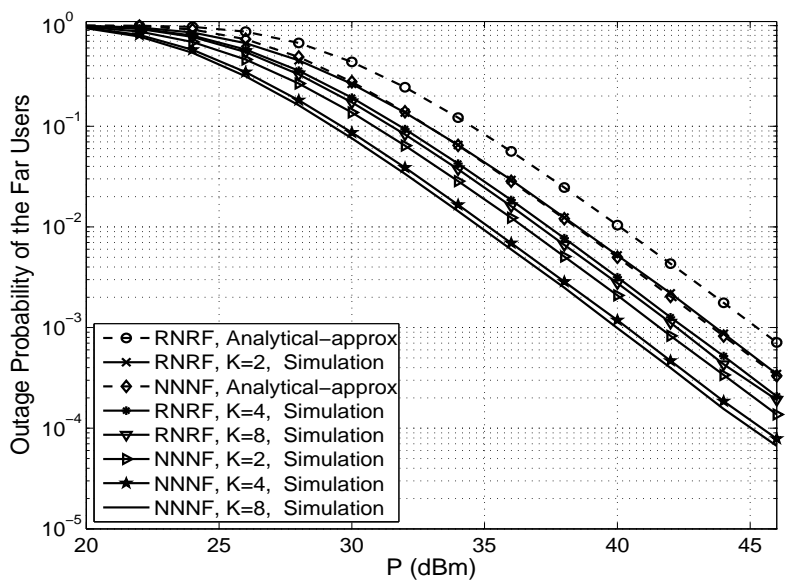

Fig. 5. Outage probability of the far users versus $P$ for TZF beamforming where $M_{T}=3$ and $M_{R}=2$.

As a result, the performance gap between RNRF and NNNF strategies increases with increasing $R_{1}$.

In Fig. 4, the outage behavior of the full-duplex and halfduplex relaying is compared for the RNRF and NNNF strategies with different levels of inter-user interference strength under the "RF chain preserved" condition [7]. In the regime of larger values of $P$, half-duplex relaying yields a better outage performance. However, full-duplex relaying is shown to yield favorable outage performances in the low-to-medium range of $P$, especially for the NNNF user selection. Interestingly, when compared to the half-duplex relaying, the full-duplex relaying can reduce the outage probability by about $63 \%$ and $55 \%$ in the NNNF and RNRF strategies, respectively, at $P=30 \mathrm{dBm}$.

Finally, Figs. 2, 3, and 4 depict that the outage probability of the near users in the full-duplex relaying shows an outage floor at high power values, for both RNRF and NNNF strategies. This is expected because the inter-user interference at the near users will be maximal with high relay transmit power, which reduces the outage performance. Sophisticated beamforming designs are capable of eliminating this floor, however, the penalty paid in the design is the additional CSI burden. 


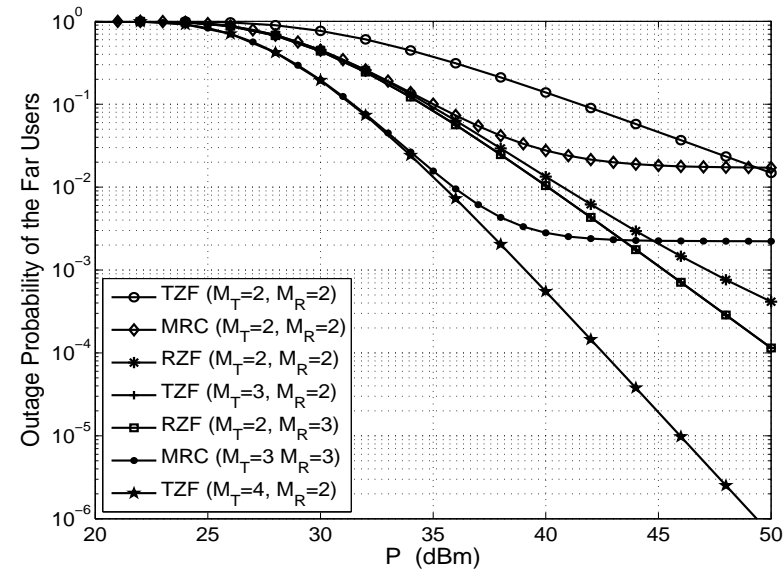

Fig. 6. Outage probability of the far users versus $P$ for the beamforming designs with different antenna configurations and RNRF user selection.

\section{B. Outage Probability of the Far Users}

Fig. 5 shows the outage probability of the far users versus $P$ with the RNRF and NNNF strategies, TZF beamforming and different number of relays, where the analytical results are based on Proposition 2 and Proposition 6. Unless otherwise stated, the values of $R_{1}, R_{2}$, and $R_{3}$ are set as $100 \mathrm{~m}, 400$ $\mathrm{m}$, and $500 \mathrm{~m}$, respectively, and $\lambda_{f}=0.0004$. It is observed that the NNNF user selection achieves a superior outage performance as compared to the RNRF user selection. Fig. 5 also shows that there is a difference between the approximate and simulation results. This is because the analytical approximations in Proposition 2 and Proposition 6 are derived under the assumption, $R_{2} \gg R_{1}$ where $\ell\left(\mathbb{R}, U_{2, i}\right) \approx \ell\left(U_{2, i}\right)$. In addition, simulation results, not shown here to avoid clutter, showed that the deviation between the analytical and simulation results decreases as either $R_{1}$ decreases or $R_{2}$ increases.

Fig. 6 shows the outage probability of the proposed beamforming schemes with different antenna configurations for the RNRF user selection. In the ZF-based beamforming schemes, since the relay is capable of canceling SI, we see that the outage probability decreases with increasing $P$. However, increasing the relay transmission power results in a strong SI in the MRC/MRT scheme, and hence the outage probability shows a floor at high SNRs. Comparing the TZF and RZF schemes, we see that the outage performances of TZF $(3,2)$ and $\operatorname{RZF}(2,3)$ (or $\operatorname{TZF}(4,2)$ and $\operatorname{RZF}(3,3)$ ) are the same. Moreover, for the case with $M_{T}=M_{R}$, RZF achieves a better performance. For the TZF with $\left(M_{T}, 2\right)$, we see that the additional transmit antenna could increase the SNR of the second hop and enhance the outage performance. However, the outage performance of RZF $\left(2, M_{R}\right)$ is less sensitive to $M_{R}$ since in the considered system, the second hop channel is more critical for the outage performance than the first hop channel. This observation shows that it is not always possible to deliver a notable performance improvement by simply increasing the total number of antennas, and therefore the configuration and beamforming design have to be carefully decided.

The far user outage probability with beamforming designs and user selection strategies for different radii, $R_{1}, R_{2}$, and $R_{3}$, is shown in Fig. 7. It can be observed from this figure that increasing $R_{3}$ (the outer radius of the far user's ring)

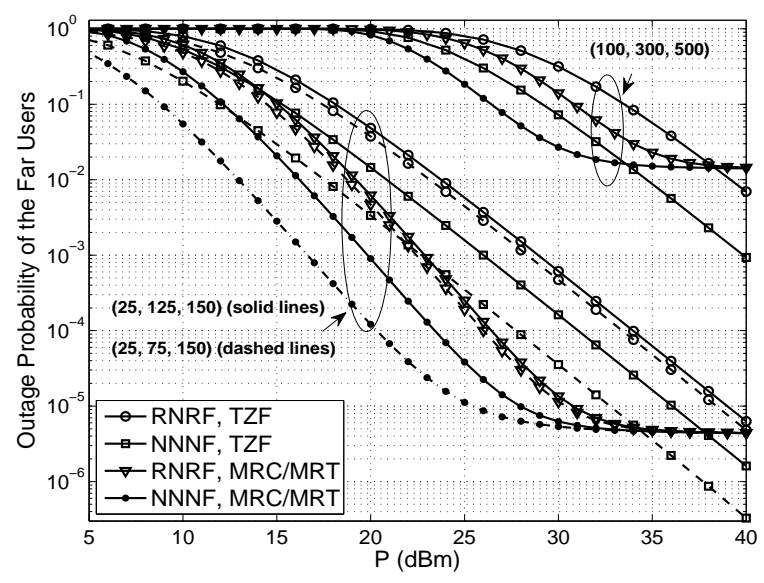

Fig. 7. Outage probability of the far users versus $P$ for different $R_{1}, R_{2}$, and $R_{3},\left(R_{1}, R_{2}, R_{3}\right)$ in meters, where $M_{T}=3$ and $M_{R}=2$.

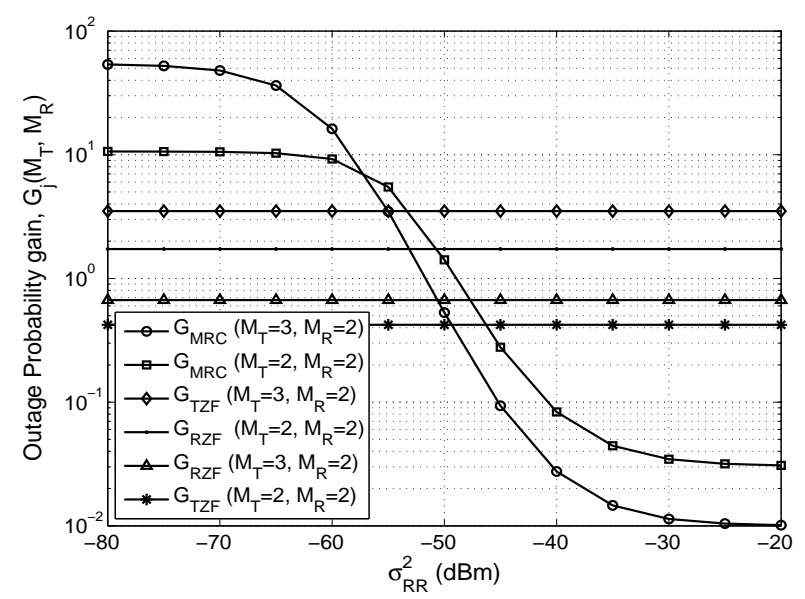

Fig. 8. Outage probability gain of the far users versus $\sigma_{R R}^{2}$ for the RNRF user selection and different beamforming designs with different antenna configurations.

degrades the outage performance of both the RNRF and NNNF strategies due to the larger path loss. The negative impact on the outage probability is more pronounced in the case of the NNNF user selection with MRC/MRT beamforming. Also, for the fixed values of $R_{1}$ and $R_{3}$ reducing $R_{2}$ can improve the NNNF outage performance, however, for the RNRF strategy, the improvement is marginal. The impact of different beamforming designs on the outage performance is more significant with the NNNF user selection. Interestingly, with the RNRF user selection, in the case of $R_{1}=25 \mathrm{~m}$, $R_{2}=125 \mathrm{~m}$ and $R_{3}=150 \mathrm{~m}$, MRC/MRT outperforms TZF in almost all transmit power regimes.

In Fig. 8, we compare the full-duplex and half-duplex relaying for different levels of SI and the RNRF user selection. More specifically, we plot the outage probability gain which is defined as $G_{\mathrm{j}}\left(M_{T}, M_{R}\right)=\frac{\mathrm{P}_{\text {out }, 2}^{\mathrm{HD}}}{P_{\text {out }, 2}^{\mathrm{j}}}, \mathrm{j} \in\{\mathrm{TZF}, \mathrm{RZF}, \mathrm{MRC}\}$ versus the SI strength, $\sigma_{R R}^{2}$. We see that the full-duplex relaying can significantly outperform its half-duplex counterpart. Nevertheless, when SI strength is low $\left(\sigma_{R R}^{2}<-53\right.$ $\mathrm{dBm})$, the gains achieved by the ZF-based designs appear to be limited when compared to the MRC/MRT scheme; e.g., $G_{\mathrm{TZF}}(3,2)=3.45$ as compared to $G_{\mathrm{MRC}}(2,2)=10$ at 


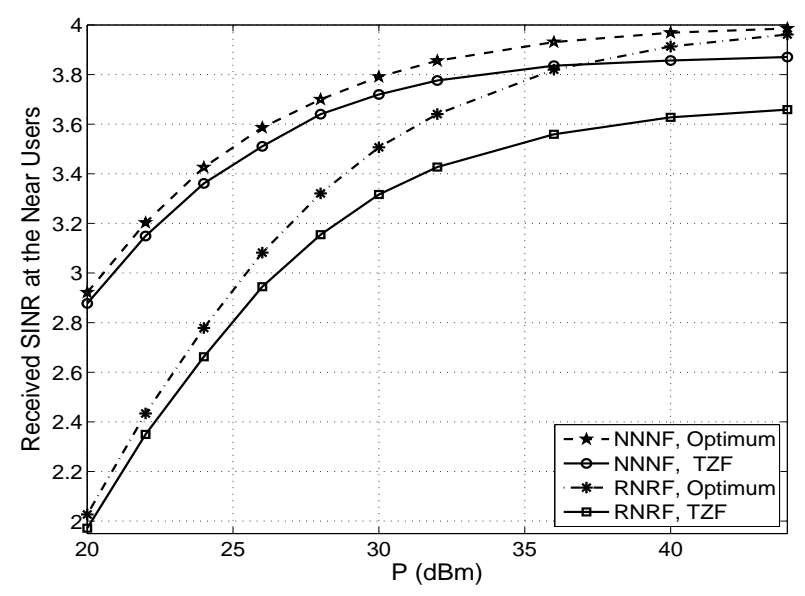

Fig. 9. The received SINR at the near users versus $P$ for different beamforming designs where $M_{T}=4$ and $M_{R}=2$.

$\sigma_{R R}^{2}=-70 \mathrm{dBm}$. In this region, $\operatorname{MRC} / \operatorname{MRT}(3,2)$ exhibits the largest gain. As observed, ZF-based designs do not suffer from SI, and hence $G_{\text {TZF }}$ and $G_{\text {RZF }}$ remain constant. On the contrary, $G_{\mathrm{MRC}}$ decreases as $\sigma_{R R}^{2}$ increases.

\section{Performance Comparison Between the Optimum and Sub- optimum Beamforming Schemes}

Fig. 9 compares the average SINR at the near users due to the optimum and TZF beamforming designs for the RNRF and NNNF user selection strategies. Since the received SINR at the near users are the same for the TZF, RZF, and MRC/MRT schemes, we only present results for the TZF scheme. Fig. 9 shows the superiority of the optimal design over TZF design, which improves with the increasing transmission power. Further, it can be observed that in the relay-assisted NOMA system with the TZF beamforming, there is a noticeable difference between the received SINR for the RNRF and NNNF user selection strategies, whereas with the optimum beamforming, RNRF converges to the NNNF at high transmit power regime. Therefore, with optimum beamforming and in the high SNR regime, the RNRF strategy provides a better performance/implementation complexity trade-off compared to its NNNF counterpart. This is a promising result since the RNRF scheme does not require the CSI knowledge of the users and provides greater fairness than NNNF. This observation reveals that the inferior performance exhibited by the RNRF in general, can be improved up to a satisfactory level when the optimum beamforming strategy is adopted.

\section{CONCLUSION}

We considered downlink NOMA transmission between an AP and two sets of users aided by a full-duplex multi-antenna relay. We proposed both optimum and suboptimal beamforming schemes and derived expressions for the outage probability of the RNRF and NNNF user selection strategies. Special cases, where closed-form expressions were possible along with bounds on the outage performance, were also presented. Our results suggest that, with suboptimal beamforming designs there is a non-negligible performance difference between the RNRF and NNNF user selection strategies, whereas in the system with optimum beamforming, the RNRF user selection performance converges to its NNNF counterpart at high transmit power regime. Moreover, NNNF user selection is more favorable than the RNRF user selection for the networks with a larger radius of the near user zone. We also showed that ZFbased beamforming significantly improves outage performance of the far users, while the MRC/MRT scheme is more efficient for scenarios with low SI interference or scenarios in which the radius of the far user's zone is large. In addition, full-duplex relaying with the proposed beamforming designs outperforms half-duplex relaying.

As for future work, it would be interesting to combine NOMA and fractional frequency reuse-based schemes to further improve the performance especially in a multi-cell network as well as to investigate the performance of various transmission schemes with a multi-antenna AP.

\section{APPENDIX A}

\section{ProOF OF PROPOSITION 1}

Let $Y_{0} \triangleq\left|\mathbf{f}_{1, i}^{T} \mathbf{w}_{t, i}^{\mathrm{ZF}}\right|^{2}$ and $Y_{1}=\left|h_{1, i}\right|^{2}$. Applying (15) and (16) into (14), the outage probability for $U_{1, i}$ can be written as

$$
\begin{aligned}
\mathrm{P}_{\text {out }, 1}^{\mathrm{TZF}}= & 1-\operatorname{Pr}\left(\frac{\rho_{s} a_{2, i} \ell\left(U_{1, i}\right) Y_{1}}{\rho_{s} a_{1, i} \ell\left(U_{1, i}\right) Y_{1}+\rho_{r} \ell\left(\mathbb{R}, U_{1, i}\right) Y_{0}+1}>\tau_{2},\right. \\
& \left.\frac{\rho_{s} a_{1, i} \ell\left(U_{1, i}\right) Y_{1}}{\rho_{r} \ell\left(\mathbb{R}, U_{1, i}\right) Y_{0}+1}>\tau_{1}\right) \\
= & \operatorname{Pr}\left(\rho_{r} \ell\left(\mathbb{R}, U_{1, i}\right) Y_{0}+1>\frac{1}{\mu} \ell\left(U_{1, i}\right) Y_{1}\right) .
\end{aligned}
$$

In (57), if $\tau_{2}>\frac{a_{2, i}}{a_{1, i}}, \mu<0$, and hence $\mathrm{P}_{\text {out }, 1}^{\mathrm{TZF}}=1$. On the other hand, when $\tau_{2} \leq \frac{a_{2, i}}{a_{1, i}}$, conditioned on $Y_{0}, \mathrm{P}_{\text {out }, 1}^{\mathrm{TZF}}$ can be expressed as

$$
\mathrm{P}_{\text {out }, 1}^{\mathrm{TZF}}=\operatorname{Pr}\left(Y_{1} \leq\left(\rho_{r} \ell\left(\mathbb{R}, U_{1, i}\right) Y_{0}+1\right) \frac{\mu}{\ell\left(U_{1, i}\right)}\right) .
$$

Note that we model the locations of the near and far users as i.i.d. points in $D_{n}$ and $D_{f}$, which are denoted by $W_{n, i}$ and $W_{f, i}$, respectively, with their corresponding pdfs $f_{W_{n, i}}\left(w_{n, i}\right)=\frac{\lambda_{n}}{\mu_{n}}=\frac{1}{\pi R_{1}^{2}}$ and $f_{W_{f, i}}\left(w_{f, i}\right)=\frac{\lambda_{f}}{\mu_{f}}=\frac{1}{\pi\left(R_{3}^{2}-R_{2}^{2}\right)}$. Therefore, (58) can be expressed as

$$
\begin{aligned}
\mathrm{P}_{\text {out }, 1}^{\mathrm{TZF}} \stackrel{(a)}{=} & \int_{D_{n}} \int_{-\pi}^{\pi} \int_{0}^{\infty}\left(1-e^{-\frac{\mu}{\ell\left(U_{1, i}\right)}\left(\rho_{r} \ell\left(\mathbb{R}, U_{1, i}\right) y+1\right)}\right) \frac{1}{q_{r}} e^{-\frac{y}{q_{r}}} \\
& \times f_{\Theta_{i}}\left(\theta_{i}\right) f_{W_{n, i}}\left(w_{n, i}\right) d y d \theta_{i} d w_{n, i} \\
= & 1-\int_{D_{n}} \int_{-\pi}^{\pi} \frac{e^{-\frac{\mu}{\ell\left(U_{1, i}\right)}}}{1+\frac{q_{r} \rho_{r} \mu}{\ell\left(U_{1, i}\right)} \ell\left(\mathbb{R}, U_{1, i}\right)} f_{\Theta_{i}}\left(\theta_{i}\right) f_{W_{n, i}}\left(w_{n, i}\right) \\
& \times d \theta_{i} d w_{n, i},
\end{aligned}
$$

where (a) follows from the fact that $Y_{0}$ and $Y_{1}$ are exponential RVs with the cdfs $F_{Y_{0}}(y)=1-e^{-y / q_{r}}$ and $F_{Y_{1}}(y)=1-$ $e^{-y}$, respectively. Substituting $f_{\Theta_{i}}\left(\theta_{i}\right)=\frac{1}{2 \pi}$ and $f_{W_{n, i}}\left(w_{n, i}\right)$ into (59), we get the desired result in (17).

\section{APPENDIX B}

\section{PROOF OF PROPOSITION 2}

Let us denote $Y_{2}=\left\|\mathbf{h}_{R}\right\|^{2}$ and $Y_{3}=\left\|\tilde{\mathbf{f}}_{2, i}\right\|^{2}$. Substituting $\tilde{\gamma}_{R}$ and $\tilde{\gamma}_{2, i}^{x_{2}, i}$ into (25), $\mathrm{P}_{\text {out }, 2}^{\mathrm{TZF}}$ can be written as 


$$
\begin{aligned}
& \mathrm{P}_{\text {out }, 2}^{\mathrm{TZF}}=\operatorname{Pr}\left(\frac{\rho_{s} a_{2, i} \ell(\mathbb{R}) Y_{2}}{\rho_{s} a_{1, i} \ell(\mathbb{R}) Y_{2}+1}<\tau_{2}\right)+ \\
& \operatorname{Pr}\left(\frac{\rho_{s} a_{2, i} \ell(\mathbb{R}) Y_{2}}{\rho_{s} a_{1, i} \ell(\mathbb{R}) Y_{2}+1}>\tau_{2}\right) \operatorname{Pr}\left(\rho_{r} \ell\left(\mathbb{R}, U_{2, i}\right) Y_{3}<\tau_{2}\right) .
\end{aligned}
$$

The RV $Y_{2}$ follows a chi-square distribution with $2 N_{\mathrm{R}}$ degreesof-freedom (DoF). Moreover, to guarantee the implementation of NOMA, the condition $\frac{a_{2, i}}{a_{1, i}} \geq \tau_{2}$ should be satisfied. Hence, $\mathrm{P}_{\text {out }, 2}^{\mathrm{TZF}}$ can be written as

$$
\begin{aligned}
\mathrm{P}_{\text {out }, 2}^{\mathrm{TZF}}= & 1-\frac{1}{\Gamma\left(N_{\mathrm{R}}\right)} \Gamma\left(N_{\mathrm{R}}, \frac{1+R_{1}^{\alpha}}{\zeta}\right)+\frac{1}{\Gamma\left(N_{\mathrm{R}}\right)} \Gamma\left(N_{\mathrm{R}}, \frac{1+R_{1}^{\alpha}}{\zeta}\right) \\
& \times \operatorname{Pr}\left(\rho_{r} \ell\left(\mathbb{R}, U_{2, i}\right) Y_{3}<\tau_{2}\right) .
\end{aligned}
$$

The next step is to compute $\operatorname{Pr}\left(\rho_{r} \ell\left(\mathbb{R}, U_{2, i}\right)^{-\alpha} Y_{3}<\tau_{2}\right)$, wherein the RV $Y_{3}$ follows a Chi-square distribution with $2\left(N_{\mathrm{T}}-1\right)$ DoF. Moreover, since $R_{2} \gg R_{1}$, we have $\ell\left(\mathbb{R}, U_{2, i}\right) \approx \ell\left(U_{2, i}\right)$ [10]. Accordingly,

$$
\begin{aligned}
& \operatorname{Pr}\left(Y_{3}<\frac{\tau_{2}}{\rho_{r} \ell\left(U_{2, i}\right)}\right)=\int_{D_{f}}\left(1-e^{-\left(\frac{\tau_{2}}{\rho_{r}}\right)\left(1+r^{\alpha}\right)} \sum_{k=0}^{N_{\mathrm{T}}-2} \frac{1}{k !}\right. \\
& \left.\quad \times\left(\frac{\tau_{2}}{\rho_{r}}\right)^{k}\left(1+r^{\alpha}\right)^{k}\right) f_{W_{f, i}}\left(w_{f, i}\right) d w_{f, i} .
\end{aligned}
$$

Applying $f_{W_{f, i}}\left(w_{f, i}\right)=\frac{1}{\pi\left(R_{3}^{2}-R_{2}^{2}\right)}$, (62) can be simplified as

$$
\begin{aligned}
\operatorname{Pr}\left(Y_{3}\right. & \left.<\frac{\tau_{2}}{\rho_{r} \ell\left(U_{2, i}\right)}\right)=1-\frac{2}{R_{3}^{2}-R_{2}^{2}} \\
\times & \int_{R_{2}}^{R_{3}}\left(e^{-\left(\frac{\tau_{2}}{\rho_{r}}\right)\left(1+r^{\alpha}\right)} \sum_{k=0}^{N_{T}-2} \frac{1}{k !}\left(\frac{\tau_{2}}{\rho_{r}}\right)^{k}\left(1+r^{\alpha}\right)^{k}\right) r d r \\
=1 & -\frac{2}{R_{3}^{2}-R_{2}^{2}} \sum_{k=0}^{N_{\text {T }}-2} \frac{1}{k !}\left(\frac{\tau_{2}}{\rho_{r}}\right)^{k} \Psi_{0},
\end{aligned}
$$

where $\Psi_{0}=\int_{R_{2}}^{R_{3}}\left(1+r^{\alpha}\right)^{k} e^{-\left(\frac{\tau_{2}}{\rho_{r}}\right)\left(1+r^{\alpha}\right)} r d r$. For an arbitrary $\alpha>2, \Psi_{0}$ is intractable. Therefore, we apply the GaussianChebyshev quadrature method to find an approximation of $\Psi_{0}$ as follows

$\Psi_{0} \approx \frac{\pi\left(R_{3}-R_{2}\right)}{2 M} \sum_{m=1}^{M} z_{m} \sqrt{1-\phi_{m}^{2}}\left(1+z_{m}^{\alpha}\right)^{k} e^{-\left(\frac{\tau_{2}}{\rho_{r}}\right)\left(1+z_{m}^{\alpha}\right)}$.

By substituting (64) into (63) and then the result into (61), we obtain (26).

\section{APPENDIX C}

PROOF OF PROPOSITION 3

Invoking (25), and substituting $\mathbf{w}_{r}^{\mathrm{MRC}}$ and $\mathbf{w}_{t, i}^{\mathrm{MRT}}$ into (5) and (10), the outage probability of the far users with the MRC/MRT scheme can be expressed as

$$
\begin{aligned}
\mathrm{P}_{\text {out }, 2}^{\mathrm{MRC}}= & \operatorname{Pr}\left(\frac{\rho_{s} a_{2, i} \ell(\mathbb{R}) Y_{2}}{\rho_{s} a_{1, i} \ell(\mathbb{R}) Y_{2}+\rho_{r} Y_{4}+1}<\tau_{2}\right) \\
& +\operatorname{Pr}\left(\frac{\rho_{s} a_{2, i} \ell(\mathbb{R}) Y_{2}}{\rho_{s} a_{1, i} \ell(\mathbb{R}) Y_{2}+\rho_{r} Y_{4}+1}>\tau_{2}\right) \\
& \times \operatorname{Pr}\left(\rho_{r} \ell\left(\mathbb{R}, U_{2, i}\right) Y_{5}<\tau_{2}\right),
\end{aligned}
$$

where $Y_{4}=\left|\mathbf{w}_{r}^{\mathrm{MRC}}{ }^{\dagger} \mathbf{H}_{R R} \mathbf{w}_{t, i}^{\mathrm{MRT}}\right|^{2}$ has an exponential distribution with parameter $\sigma_{R R}^{2}$ and $Y_{5}=\left\|\mathbf{f}_{2, i}\right\|^{2}$ follows a Chisquare distribution with $2 N_{\mathrm{T}}$ DoF. $\mathrm{P}_{\mathrm{out}, 2}^{\mathrm{MRC}}$ can be re-expressed as

$$
\begin{aligned}
\mathrm{P}_{\text {out }, 2}^{\mathrm{MRC}}= & 1-\operatorname{Pr}\left(\frac{\rho_{s} a_{2, i} \ell(\mathbb{R}) Y_{2}}{\rho_{s} a_{1, i} \ell(\mathbb{R}) Y_{2}+\rho_{r} Y_{4}+1}>\tau_{2}\right) \\
& \times \operatorname{Pr}\left(\rho_{r} \ell\left(\mathbb{R}, U_{2, i}\right) Y_{5}>\tau_{2}\right) .
\end{aligned}
$$

Using similar steps as in Proposition 2 and the approximation $\ell\left(\mathbb{R}, U_{2, i}\right) \approx \ell\left(U_{2, i}\right)$, we can write

$$
\begin{array}{r}
\operatorname{Pr}\left(\rho_{r} \ell\left(U_{2, i}\right) Y_{5}>\tau_{2}\right)=\frac{\pi}{M\left(R_{3}+R_{2}\right)} \sum_{k=0}^{N_{\mathrm{T}}-1} \frac{1}{k !}\left(\frac{\tau_{2}}{\rho_{r}}\right)^{k} \\
\times \sum_{m=1}^{M} z_{m} \sqrt{1-\phi_{m}^{2}}\left(1+z_{m}^{\alpha}\right)^{k} e^{-\left(\frac{\tau_{2}}{\rho_{r}}\right)\left(1+z_{m}^{\alpha}\right)^{k}} .
\end{array}
$$

Thus, the remaining task is to compute $I \triangleq$ $\operatorname{Pr}\left(\frac{\rho_{s} a_{2, i} \ell(\mathbb{R}) Y_{2}}{\rho_{s} a_{1, i} \ell(\mathbb{R}) Y_{2}+\rho_{r} Y_{4}+1}>\tau_{2}\right)$ which can be expressed as

$$
\begin{aligned}
I= & \int_{\frac{1}{\zeta \ell(\mathbb{R})}}^{\infty}\left(1-e^{-\frac{\zeta \ell(\mathbb{R})}{\rho_{r} \sigma_{R R}^{2}}}\right) f_{Y_{2}}(y) d y \\
= & \frac{1}{\Gamma\left(N_{R}\right)} \Gamma\left(N_{R}, \frac{1}{\zeta \ell(\mathbb{R})}\right)-\frac{e^{\frac{1}{\rho_{r} \sigma_{R R}^{2}}}}{\Gamma\left(N_{R}\right)}\left(\frac{\zeta \ell(\mathbb{R})}{\rho_{r} \sigma_{R R}^{2}}+1\right)^{-N_{R}} \\
& \times \Gamma\left(N_{R}, \frac{1}{\rho_{r} \sigma_{R R}^{2}}+\frac{1}{\zeta \ell(\mathbb{R})}\right)
\end{aligned}
$$

where $f_{Y_{2}}(y)=\frac{y^{N_{R}-1} e^{-y}}{\Gamma\left(N_{R}\right)}$ is the pdf of the RV $Y_{2}$ and [30, Eq. (3.351.2)] was used to simplify the integral. Finally, combining (67) and (68), we obtain (31).

\section{APPENDIX D}

\section{PROOF OF PROPOSITION 4}

Substituting (34) and (35), into (14) we obtain

$$
\begin{aligned}
\mathrm{P}_{\text {out }, 1}^{\mathrm{HD}}= & -\operatorname{Pr}\left(\frac{\rho_{s} a_{2, i} \ell\left(U_{1, i}\right) Y_{1}}{\rho_{s} a_{1, i} \ell\left(U_{1, i}\right) Y_{1}+1}>\tau_{2}^{\mathrm{HD}},\right. \\
& \left.\rho_{s} a_{1, i} \ell\left(U_{1, i}\right) Y_{1}>\tau_{1}^{\mathrm{HD}}\right),
\end{aligned}
$$

which can be written as

$$
\mathrm{P}_{\text {out }, 1}^{\mathrm{HD}}=1-\frac{2}{R_{1}^{2}} \int_{0}^{R_{1}} e^{-\mu^{\mathrm{HD}}\left(1+r^{\alpha}\right)} r d r
$$

for $\tau_{2}^{\mathrm{HD}} \leq \frac{a_{2, i}}{a_{1, i}}$. Applying the gaussian-Chebyshev quadrature approximation into (70), the outage probability of $U_{1, i}$ with the half-duplex relaying can be expressed as (36) if $\tau_{2}^{\mathrm{HD}} \leq$ $\frac{a_{2, i}}{a_{1, i}}$. Otherwise, $\mathrm{P}_{\text {out }, 1}^{\mathrm{HD}}=1$. Moreover, plugging (10) and (33) into (25), $\mathrm{P}_{\text {out }, 2}^{\mathrm{HD}}$ can be expressed as

$$
\begin{aligned}
& \mathrm{P}_{\text {out }, 2}^{\mathrm{HD}}=\operatorname{Pr}\left(\frac{\rho_{s} a_{2, i} \ell(\mathbb{R}) Y_{2}}{\rho_{s} a_{1, i} \ell(\mathbb{R}) Y_{2}+1}<\tau_{2}^{\mathrm{HD}}\right)+ \\
& \operatorname{Pr}\left(\frac{\rho_{s} a_{2, i} \ell(\mathbb{R}) Y_{2}}{\rho_{s} a_{1, i} \ell(\mathbb{R}) Y_{2}+1}>\tau_{2}^{\mathrm{HD}}\right) \operatorname{Pr}\left(\rho_{r} \ell\left(\mathbb{R}, U_{2, i}\right) Y_{5}<\tau_{2}^{\mathrm{HD}}\right),
\end{aligned}
$$

where $Y_{5}=\left\|\mathbf{f}_{2, i}\right\|^{2}$ follows the Chi-square distribution with $2 N_{\mathrm{T}}$ DoF. Using similar steps as in Proposition 2, we obtain (37). 


\section{APPENDIX E}

\section{PROOF OF PROPOSITION 5}

Similar to (58), $\mathrm{P}_{\text {out }, 1^{\star}}^{\mathrm{TZF}}$ for $U_{1, i}^{\star}$ can be written as $\mathrm{P}_{\text {out }, 1^{\star}}^{\mathrm{TZF}}=\operatorname{Pr}\left(Y_{1} \leq\left(\rho_{r} \ell\left(\mathbb{R}, U_{1, i}^{\star}\right) Y_{0}+1\right) \frac{\mu}{\ell\left(U_{1, i}^{\star}\right)} \mid Y_{0}, N_{U_{1}} \geq 1\right)$.

By following similar steps as in the derivation of (59), $\mathrm{P}_{\text {out }, 1^{\star}}^{\mathrm{TZF}}$ for $U_{1, i}^{\star}$ can be written as

$$
\begin{aligned}
\mathrm{P}_{\text {out }, 1^{\star}}^{\mathrm{TZF}} & =\frac{1}{2 \pi} \int_{0}^{R_{1}} \int_{\pi}^{\pi}\left(1-\frac{e^{-\mu\left(1+r^{\alpha}\right)}}{1+\frac{q_{r} \rho_{r} \mu\left(1+r^{\alpha}\right)}{1+\left(R_{1}^{2}+r^{2}-2 r R_{1} \cos \left(\theta_{r}-\theta_{i}\right)\right)^{\frac{\alpha}{2}}}}\right) \\
& \times f_{n^{*}}(r) d \theta_{i} d r
\end{aligned}
$$

where $f_{n^{*}}(r)$ is the pdf of the shortest distance from $U_{1, i}^{\star}$ to the AP, which is given by [10]

$$
f_{n^{*}}(r)=v_{n} r e^{-\pi \lambda_{n} r^{2}} .
$$

Substituting (74) into (73), the proposition is proved.

\section{APPENDIX F \\ PROOF OF PROPOSITION 6}

The outage probability of $U_{2, i}^{\star}$ can be expressed as

$$
\begin{aligned}
\mathrm{P}_{\text {out }, 2^{\star}}^{\mathrm{TZF}}= & \operatorname{Pr}\left(\frac{\rho_{s} a_{2, i} \ell(\mathbb{R}) Y_{2}}{\rho_{s} a_{1, i} \ell(\mathbb{R}) Y_{2}+1}<\tau_{2} \mid N_{U_{2}} \geq 1\right) \\
& +\operatorname{Pr}\left(\frac{\rho_{s} a_{2, i} \ell(\mathbb{R}) Y_{2}}{\rho_{s} a_{1, i} \ell(\mathbb{R}) Y_{2}+1}>\tau_{2} \mid N_{U_{2}} \geq 1\right) \\
& \times \operatorname{Pr}\left(\rho_{r} \ell\left(\mathbb{R}, U_{2, i}^{\star}\right) Y_{3}<\tau_{2} \mid N_{U_{2}} \geq 1\right) .
\end{aligned}
$$

Since $R_{2} \gg R_{1}$, we can approximate $\ell\left(\mathbb{R}, U_{2, i}^{\star}\right) \approx \ell\left(U_{2, i}^{\star}\right)$ and $\mathrm{P}_{\text {out }, 2^{\star}}^{\mathrm{TZF}}$ can be evaluated as

$$
\begin{aligned}
\mathrm{P}_{\text {out }, 2^{\star}}^{\mathrm{TZF}}= & 1-\frac{1}{\Gamma\left(N_{\mathrm{R}}\right)} \Gamma\left(N_{\mathrm{R}}, \frac{1+R_{1}^{\alpha}}{\zeta}\right)+\frac{1}{\Gamma\left(N_{\mathrm{R}}\right)} \Gamma\left(N_{\mathrm{R}}, \frac{1+R_{1}^{\alpha}}{\zeta}\right) \\
& \times \operatorname{Pr}\left(Y_{3}<\frac{\tau_{2}}{\rho_{r} \ell\left(U_{2, i}^{\star}\right)} \mid N_{U_{2}} \geq 1\right) .
\end{aligned}
$$

We note that $Y_{3}$ is a Chi-square distributed $\mathrm{RV}$ with $2\left(N_{\mathrm{T}}-1\right)$ DoF, and thus

$$
\begin{aligned}
F_{Y_{3}}\left(\frac{\tau_{2}}{\rho_{r} \ell\left(U_{2, i}^{\star}\right)}\right)= & \int_{R_{2}}^{R_{3}}\left(1-e^{-\left(\frac{\tau_{2}}{\rho_{r}}\right)\left(1+r^{\alpha}\right)} \sum_{k=0}^{N_{\mathrm{T}}-2} \frac{1}{k !}\left(\frac{\tau_{2}}{\rho_{r}}\right)^{k}\right. \\
& \left.\times\left(1+r^{\alpha}\right)^{k}\right) f_{f}^{*}(r) d r,
\end{aligned}
$$

where $f_{f}^{*}(r)=v_{f} r e^{-\pi \lambda_{f}\left(r^{2}-R_{2}^{2}\right)}$ [10] is the pdf of the nearest $U_{2, i}^{\star}$. Next, substituting $f_{f}^{*}(r)$ into (77), we obtain $F_{Y_{3}}\left(\frac{\tau_{2}}{\rho_{r} \ell\left(U_{2, i}^{*}\right)}\right)=1-v_{f} e^{\pi \lambda_{f} R_{2}^{2}} \sum_{k=0}^{N_{\mathrm{T}}-2} \frac{1}{k !}\left(\frac{\tau_{2}}{\rho_{r}}\right)^{k} \Psi_{1}$, where $\Psi_{1}=\int_{R_{2}}^{R_{3}} e^{-\left(\frac{\tau_{2}}{\rho_{r}}+\frac{\tau_{2}}{\rho_{r}} r^{\alpha}+\pi \lambda_{f} r^{2}\right)} \times\left(1+r^{\alpha}\right)^{k} r d r$. An exact evaluation of $\Psi$ is mathematically intractable. Hence, we use the Gaussian-Chebyshev quadrature method to find an approximation as

$$
\begin{aligned}
\Psi_{1} \approx & \frac{\pi\left(R_{3}-R_{2}\right)}{2 M} \sum_{m=1}^{M} z_{m} \sqrt{1-\phi_{m}^{2}}\left(1+z_{m}^{\alpha}\right)^{k} \\
& \times e^{-\left(\frac{\tau_{2}}{\rho_{r}}+\frac{\tau_{2}}{\rho_{r}} z_{m}^{\alpha}+\pi \lambda_{f} z_{m}^{2}\right)} .
\end{aligned}
$$

Substituting (78) into $F_{Y_{3}}\left(\frac{\tau_{2}}{\rho_{r} \ell\left(U_{2, i}^{*}\right)}\right)$ and next the result into (76), we arrive at the desired result.

\section{REFERENCES}

[1] Z. Mobini, M. Mohammadi, H. A. Suraweera, and Z. Ding, "Fullduplex multi-antenna relay assisted cooperative non-orthogonal multiple access," in Proc. IEEE Global Commun. Conf. (GLOBECOM'17), Singapore, Dec. 2017, pp. 1-7.

[2] Y. Saito, Y. Kishiyama, A. Benjebbour, T. Nakamura, A. Li, and K. Higuchi, "Non-orthogonal multiple access (NOMA) for cellular future radio access," in Proc. IEEE Veh. Technol. Conf. (VTC'13), Dresden, Germany, June 2013, pp. 1-5.

[3] Z. Ding, Y. Liu, J. Choi, Q. Sun, M. Elkashlan, C.-L. I, and H. V. Poor, "Application of non-orthogonal multiple access in LTE and 5G networks," IEEE Commun. Mag., vol. 55, pp. 185-191, Feb. 2017.

[4] A. Sabharwal, P. Schniter, D. Guo, D. W. Bliss, S. Rangarajan, and R. Wichman, "In-band full-duplex wireless: Challenges and opportunities," IEEE J. Sel. Areas Commun., vol. 32, pp. 1637-1652, Sep. 2014.

[5] T. Riihonen, S. Werner, and R. Wichman, "Mitigation of loopback selfinterference in full-duplex MIMO relays," IEEE Trans. Signal Process. vol. 59, pp. 5983-5993, Dec. 2011.

[6] M. Duarte, C. Dick, and A. Sabharwal, "Experiment-driven characterization of full-duplex wireless systems," IEEE Trans. Wireless Commun., vol. 11, pp. 4296-4307, Dec. 2012.

[7] H. Q. Ngo, H. A. Suraweera, M. Matthaiou, and E. G. Larsson, "Multipair full-duplex relaying with massive arrays and linear processing," IEEE J. Sel. Areas Commun., vol. 32, pp. 1721-1737, Sep. 2014.

[8] Z. Ding, M. Peng, and H. V. Poor, "Cooperative non-orthogonal multiple access in 5G systems," IEEE Commun. Lett., vol. 19, pp. 1462-1465, Aug. 2015.

[9] Y. Xu, C. Shen, Z. Ding, X. Sun, S. Yan, G. Zhu, and Z. Zhong, "Joint beamforming and power splitting control in downlink cooperative SWIPT NOMA systems," IEEE Trans. Signal Process., vol. 65, pp. 4874-4886, June 2017.

[10] Y. Liu, Z. Ding, M. Elkashlan, and H. V. Poor, "Cooperative nonorthogonal multiple access with simultaneous wireless information and power transfer," IEEE J. Sel. Areas Commun., vol. 34, pp. 938-953, Apr. 2016.

[11] J. Men and J. Ge, "Non-orthogonal multiple access for multiple-antenna relaying networks," IEEE Commun. Lett., vol. 19, pp. 1686-1689, Oct. 2015.

[12] J. B. Kim and I. H. Lee, "Capacity analysis of cooperative relaying systems using non-orthogonal multiple access," IEEE Commun. Lett., vol. 19, pp. 1949-1952, Nov. 2015.

[13] — "Non-orthogonal multiple access in coordinated direct and relay transmission," IEEE Commun. Lett., vol. 19, pp. 2037-2040, Nov. 2015.

[14] X. Liang, Y. Wu, D. W. K. Ng, Y. Zuo, S. Jin, and H. Zhu, "Outage performance for cooperative NOMA transmission with an AF relay," IEEE Commun. Lett., vol. 21, pp. 2428-2431, Nov. 2017.

[15] M. Xu, F. Ji, M. Wen, and W. Duan, "Novel receiver design for the cooperative relaying system with non-orthogonal multiple access," IEEE Commun. Lett., vol. 20, pp. 1679-1682, Aug. 2016.

[16] D. Wan, M. Wen, H. Yu, Y. Liu, F. Ji, and F. Chen, "Non-orthogonal multiple access for dual-hop decode-and-forward relaying," in Proc. IEEE Global Commun. Conf. (GLOBECOM'16), Washington, DC, Dec. 2016, pp. 1-6.

[17] H. Sun, Q. Wang, R. Q. Hu, and Y. Qian, "Outage probability study in a NOMA relay system," in Proc. IEEE Wireless Commun. and Networking Conf. (WCNC'17), San Francisco, CA, Mar. 2017, pp. 1-6.

[18] Z. Ding, H. Dai, and H. V. Poor, "Relay selection for cooperative NOMA," IEEE Wireless Commun. Lett., vol. 5, pp. 416-419, Aug. 2016.

[19] S. Lee, D. B. da Costa, Q. T. Vien, T. Q. Duong, and R. T. de Sousa, "Non-orthogonal multiple access schemes with partial relay selection," IET Commun., vol. 11, pp. 846-854, May 2017.

[20] S. Zhang, B. Di, L. Song, and Y. Li, "Sub-channel and power allocation for non-orthogonal multiple access relay networks with amplify-andforward protocol," IEEE Trans. Wireless Commun., vol. 16, pp. 22492261, Apr. 2017.

[21] C. Xue, Q. Zhang, Q. Li, and J. Qin, "Joint power allocation and relay beamforming in non-orthogonal multiple access amplify-and-forward relay networks," IEEE Trans. Veh. Technol., vol. 66, pp. 7558-7562, Aug. 2017.

[22] Z. Yang, Z. Ding, P. Fan, and N. Al-Dhahir, "The impact of power allocation on cooperative non-orthogonal multiple access networks with SWIPT," IEEE Trans. Wireless Commun., vol. 16, pp. 4332-4343, May 2017.

[23] M. S. Elbamby, M. Bennis, W. Saad, M. Debbah, and M. Latva-aho, "Resource optimization and power allocation in in-band full duplex 
(IBFD)-enabled non-orthogonal multiple access networks," IEEE J. Sel. Areas Commun., vol. PP, pp. 1-1, 2017.

[24] Y. Sun, D. W. K. Ng, Z. Ding, and R. Schober, "Optimal joint power and subcarrier allocation for full-duplex multicarrier non-orthogonal multiple access systems," IEEE Trans. Commun., vol. 65, pp. 1077-1091, Mar. 2017.

[25] Z. Zhang, Z. Ma, M. Xiao, Z. Ding, and P. Fan, "Full-duplex device-todevice-aided cooperative non-orthogonal multiple access," IEEE Trans. Veh. Technol., vol. 66, pp. 4467-4471, May 2017.

[26] C. Zhong and Z. Zhang, "Non-orthogonal multiple access with cooperative full-duplex relaying," IEEE Commun. Lett., vol. 20, pp. 2478-2481, Dec. 2016.

[27] X. Yue, Y. Liu, S. Kang, A. Nallanathan, and Z. Ding, "Exploiting full/half-duplex user relaying in NOMA systems," IEEE Trans. Commun., vol. 66, pp. 560-575, Feb. 2018.

[28] Z. Ding, P. Fan, and H. V. Poor, "On the coexistence between full-duplex and NOMA," IEEE Wireless Commun. Lett., pp. 1-1, 2018.

[29] A. Asadi and V. Mancuso, "A survey on opportunistic scheduling in wireless communications," IEEE Commun. Surv. Tutorial., vol. 15, pp. 1671-1688, Fourth quarter 2013.

[30] I. S. Gradshteyn and I. M. Ryzhik, Table of Integrals, Series and Products, 7th ed. San Diego, CA: Academic Press, 2007.

[31] W. Shin, M. Vaezi, B. Lee, D. J. Love, J. Lee, and H. V. Poor, "Nonorthogonal multiple access in multi-cell networks: Theory, performance, and practical challenges," IEEE Commun. Mag., vol. 55, pp. 176-183, Oct. 2017.

[32] W. Guo and T. O'Farrell, "Relay deployment in cellular networks: Planning and optimization," IEEE J. Sel. Areas Commun., vol. 31, pp. 1597-1606, Aug. 2013.

[33] L. Wang, K. Wong, R. W. Heath, and J. Yuan, "Wireless powered dense cellular networks: How many small cells do we need?" IEEE J. Sel. Areas Commun., vol. 35, pp. 2010-2024, Sep. 2017.

[34] S. R. T. A. T. A. G. I. Z. K. I. R. O. H. K. A. P. S. Sun, T. S. Rappaport and J. Järveläinen, "Propagation path loss models for 5G urban micro and macro-cellular scenarios," in Proc. IEEE Veh. Tech. Conf. (VTC Spring'16), Nanjing, China, May 2016, pp. 1-5.

[35] H. A. Suraweera, I. Krikidis, G. Zheng, C. Yuen, and P. J. Smith, "Lowcomplexity end-to-end performance optimization in MIMO full-duplex relay systems," IEEE Trans. Wireless Commun., vol. 13, pp. 913-927, Jan. 2014.

[36] M. Mohammadi, B. K. Chalise, H. A. Suraweera, C. Zhong, G. Zheng, and I. Krikidis, "Throughput analysis and optimization of wirelesspowered multiple antenna full-duplex relay systems," IEEE Trans. Commun., vol. 64, pp. 1769-1785, Apr. 2016.

[37] F. B. Hildebrand, Introduction to Numerical Analysis. New York, USA: Dover, 1987.

[38] W. W. Hager, "Updating the inverse of a matrix," SIAM Rev, vol. 31, pp. 221-239, 1989.

[39] T. R. G. . v. M. . "Evolved Universal Terrestrial Radio Access E-UTRA; Further advancements for E-UTRA physical layer aspects, "3GPP.

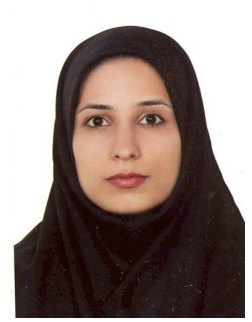

Zahra Mobini (S'09, M'15) received the B.S. degree in electrical engineering from Isfahan University of Technology, Isfahan, Iran, in 2006, and the M.S and Ph.D. degrees, both in electrical engineering, from the M. A. University of Technology and K. N. Toosi University of Technology, Tehran, Iran, respectively. From November 2010 to November 2011, she was a Visiting Researcher at the Research School of Engineering, Australian National University, Canberra, ACT, Australia. She is currently an Assistant Professor with the Faculty of Engineering, Shahrekord University, Shahrekord, Iran. Her research interests include wireless communication systems, cooperative networks, and network coding.

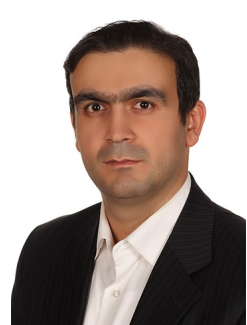

Mohammadali Mohammadi (S'09, M'15) received the B.S. degree in electrical engineering from Isfahan University of Technology, Isfahan, Iran, in 2005, and the M.S. and Ph.D. degrees in electrical engineering from K. N. Toosi University of Technology, Tehran, Iran in 2007 and 2012, respectively. From November 2010 to November 2011, he was a visiting researcher in the Research School of Engineering, the Australian National University, Australia, working on cooperative networks. He is currently an assistant professor in the Faculty of Engineering, Shahrekord University, Iran. His main research interests include cooperative communications, energy harvesting and Green communications, full-duplex communications and stochastic geometry.

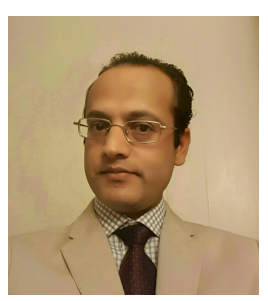

Batu K. Chalise received the M.S. and Ph.D. degrees in Electrical Engineering from the University of Duisburg-Essen, Germany. He is currently an Assistant Professor in the Department of Electrical and Computer Engineering, New York Institute of Technology, NY, USA. He was a Visiting Assistant Professor with the Department of Electrical Engineering and Computer Science, Cleveland State University, $\mathrm{OH}$, from 2015 to 2017 . He was a wireless System Research Engineer with ArrayComm, San Jose, CA from 2013 to 2015 and a Postdoctoral Research Fellow with the Center for Advanced Communications, Villanova University, PA, from 2010 to 2013. He has also held various research and teaching positions with the Catholic University of Louvain, Belgium, and University of Duisburg-Essen, Germany.

His research interests include signal processing for wireless and radar communications, wireless sensor networks, smart systems, and machine learning. He was a recipient of the U.S. Air Force Laboratory Summer Faculty Research Fellowship in 2016.

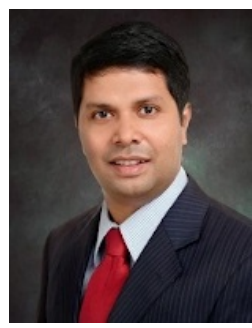

Himal A. Suraweera (S'04, M'07, SM'15) received the B.Sc. degree (Hons.) in engineering from the University of Peradeniya, Sri Lanka, in 2001, and the Ph.D. degree from Monash University, Australia, in 2007. Currently, he is a Senior Lecturer with the Department of Electrical and Electronic Engineering, University of Peradeniya.

His research interests include $5 \mathrm{G}$ networks, cooperative communications, massive MIMO and wireless energy harvesting. He was a recipient of the IEEE Communications Society Leonard G. Abraham Prize in 2017. He is an Editor of the IEEE TRANSACTIONS ON WIRELESS COMMUNICATIONS, the IEEE TRANSACTIONS ON COMMUNICATIONS, and the IEEE TRANSACTIONS ON GREEN COMMUNICATIONS AND NETWORKING. 


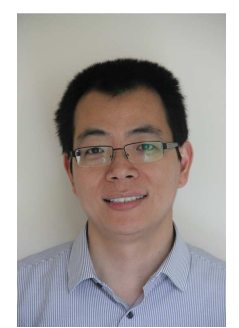

Zhiguo Ding (S'03-M'05) received his B.Eng in Electrical Engineering from the Beijing University of Posts and Telecommunications in 2000, and the $\mathrm{Ph} . \mathrm{D}$ degree in Electrical Engineering from Imperial College London in 2005.

From Jul. 2005 to Apr. 2018, he was working in Queen's University Belfast, Imperial College, Newcastle University and Lancaster University. Since Apr. 2018, he has been with the University of Manchester as a Professor in Communications. From Oct. 2012 to Sept. 2018, he has also been an academic visitor in Princeton University.

Dr Ding' research interests are 5G networks, game theory, cooperative and energy harvesting networks and statistical signal processing.

$\mathrm{He}$ is serving as an Editor for IEEE Transactions on Communications, IEEE Transactions on Vehicular Technology, and Journal of Wireless Communications and Mobile Computing, and was an Editor for IEEE Wireless Communication Letters, IEEE Communication Letters from 2013 to 2016.

He received the best paper award in IET ICWMC-2009 and IEEE WCSP2014, the EU Marie Curie Fellowship 2012-2014, the Top IEEE TVT Editor 2017, IEEE Heinrich Hertz Award 2018 and the IEEE Jack Neubauer Memorial Award 2018. 